FedUni ResearchOnline

https://researchonline.federation.edu.au

This is the author's accepted version of the following publication:

Kruger, A., López, M. (2012) Stationarity and regularity of infinite collections of sets. Applications to infinitely contstrained optimization. Journal of Optimiation Theory and Applications, 155(2), 390-416.

The version displayed here may differ from the final published version.

The final publication is available at:

https://doi.org/10.1007/s10957-012-0086-6

Copyright $($ 2012, Springer Science+Business Media LLC. 


\title{
Stationarity and Regularity of Infinite Collections of Sets. Applications to Infinitely Constrained Optimization
}

\author{
Alexander Y. Kruger · Marco A. López
}

Communicated by Michel Théra

Received: date / Accepted: date

\begin{abstract}
This article continues the investigation of stationarity and regularity properties of infinite collections of sets in a Banach space started in Kruger and López: J Optim. Theory Appl. 154(2) (2012), and is mainly focused on the application of the stationarity criteria to infinitely constrained optimization problems. We consider several settings of optimization problems, which involve (explicitly or implicitly) infinite collections of sets and deduce for them necessary conditions characterizing stationarity in terms of dual space elements - normals and/or subdifferentials.
\end{abstract}

Keywords Subdifferential · Normal cone · Optimality · Extremality · Stationarity · Regularity · Extremal principle $\cdot$ Asplund space $\cdot$ Infinitely constrained optimization

Mathematics Subject Classification (2000) 49J52 - 49J53 $\cdot 49 \mathrm{~K} 27 \cdot 90 \mathrm{C} 34$

The research was partially supported by the Australian Research Council, Project DP110102011 and by the Spanish MTM2008-06695-C03(01).

The first author is grateful to the University of Alicante for support and hospitality during his stay there in June 2011.

The authors wish to thank Michel Théra and the anonymous referees for the careful reading of the paper and valuable comments and suggestions.

A. Y. Kruger $(\varangle)$

Centre for Informatics and Applied Optimization, School of Science, Information Technology and Engineering, University of Ballarat, POB 663, Ballarat, Vic, 3350, Australia

E-mail: a.kruger@ballarat.edu.au

M. A. López

Department of Statistics and Operations Research, University of Alicante, 03080 Alicante, Spain

E-mail: marco.antonio@ua.es 


\section{Introduction}

This research is mainly motivated by applications to problems of infinite and semi-infinite programming, i.e., optimization problems with infinitely many constraints which have attracted in recent years considerable attention; see, e.g., $[1-23]$.

In numerous publications, the authors develop numerical methods and investigate various stability and regularity issues. Several articles are devoted to optimality conditions $[7,9,12,16,17,19,20,22,23]$.

Out of the convex scenario, a general optimization problem involving extended-real-valued lower semicontinuous functions, is approached in [20] by applying some tangential extremal principles and related calculus rules for infinite intersections. Assuming that the functions involved in the setting of the problem are locally Lipschitz continuous around a local solution and certain constraint qualifications (CHIP and SQC) are satisfied, asymptotic-type KKT conditions are derived in [20, Theorem 4.5] for both the upper and lower subdifferential of the objective function. Ordinary (non-asymptotic) KKT conditions are also obtained under an additional constraint qualification (called SCC).

Another approach to this topic based on variational analysis is suggested in [23]. In this paper, the authors consider a finite-dimensional infinitely-constrained optimization problem with a compact index set. A general Lagrange multiplier rule in terms of the Clarke subdifferentials and coderivatives is derived. This rule does not require any constraint qualification, but when the authors introduce the so-called $G C Q$ constraint qualification (extending to the nonsmooth setting the standard Mangasarian-Fromovitz constraint qualification), they obtain Lagrange multiplier rules in standard form, as well as standard KKT-type conditions if the constraints are additionally assumed convex.

This article continues the investigation of stationarity and regularity properties of infinite collections of sets in a Banach space started in [24] and is mainly focused on the application of the criteria from [24] to infinitely constrained optimization problems. We consider several settings of optimization problems which involve (explicitly or implicitly) infinite collections of sets and deduce for them necessary conditions characterizing stationarity in terms of dual space elements - normals or subdifferentials.

The plan of the paper is as follows. After introducing in Section 2 some basic notation we summarize in Section 3 the definitions and criteria of stationarity and regularity for infinite collections of sets from [24]. Two groups of definitions are presented: with and without a gauge function which restricts the size of finite subcollections involved in the definitions. All the properties in the second group (without a gauge function) can be defined in terms of certain constants characterizing the mutual arrangement of the sets in space. The main result is given by Theorem 3.1, which establishes the relationship between primal and dual space approximate stationarity properties. It can be considered as an extension of the Extremal principle [25-27].

In Section 4, we recall several intersection rules for Fréchet normals to infinite intersections in Asplund spaces developed in [24]. Besides the general form of the intersection rule, we formulate also its normal form under the assumption of Fréchet normal regularity of the collection of sets from Section 3. 
In Section 5, the intersection rules from Section 4 are applied to developing maximum rules for Fréchet subdifferentials. We consider the supremum function and prove several representations of its Fréchet subgradients in terms of Fréchet subgradients of the elementary functions involved in the definition.

The final Section 6 is devoted to necessary conditions of stationarity. We consider minimax problems with possibly infinite index set and two types of constrained optimization problems with infinitely many constraints given by either arbitrary sets or inequalities. Several kinds of primal space stationarity properties are defined and characterized in terms of Fréchet normals and subdifferentials. The Fréchet normal constraint qualification is introduced.

In the nonsmooth context, different alternatives to the Fréchet normal constraint qualification can be found in the literature, mainly in the convex setting. For instance, in [7], necessary optimality conditions are established for broad classes of semi-infinite programs where the cost function is a lower semicontinuous function on a Banach space, the feasible set is given by a parameterized system of infinitely many linear inequalities, and the parameter is a bounded function which defines a perturbation of the right-hand side term. Besides the calculus rules of the involved subdifferentials of the cost function, a crucial role in deriving these optimality conditions is played by the coderivative of the feasible set mapping, which was characterized in [6] entirely in terms of the initial data. The optimality conditions in this paper are established in asymptotic form, involving the weak* closure of the so-called second moment cone, while they are presented in an extended Karush-Kuhn-Tucker (KKT) form under some closedness conditions (Farkas-Minkowski type constraint qualification).

\section{Preliminaries}

Our basic notation is standard; see $[26,28]$. Throughout the paper, $X$ is a Banach space (although the definitions are valid in a normed linear space). Its topological dual is denoted $X^{*}$, while $\langle\cdot, \cdot\rangle$ denotes the bilinear form defining the pairing between $X$ and $X^{*}$. The closed unit balls in a normed space and its dual are denoted by $\mathbb{B}$ and $\mathbb{B}^{*}$, respectively. $B_{\delta}(x)$ denotes the closed ball with radius $\delta$ and center $x$.

We say that a set $\Omega \subset X$ is locally closed near $\bar{x} \in \Omega$ iff $\Omega \cap U$ is closed in $X$ for some closed neighborhood $U$ of $\bar{x}$. Given a set $I$ of indices, its cardinality (the number of elements in $I$ ) is denoted $|I|$. Functions $f_{i}(i \in I)$ are said to be uniformly Lipschitz near $\bar{x}$ iff they are Lipschitz continuous in some common neighborhood of $\bar{x}$ with the same modulus.

If $f: X \rightarrow \mathbb{R}_{\infty}:=\mathbb{R} \cup\{+\infty\}, x \in X$, and $f(x)<\infty$, then

$$
\partial^{F} f(x):=\left\{x^{*} \in X^{*} \mid \liminf _{u \rightarrow x} \frac{f(u)-f(x)-\left\langle x^{*}, u-x\right\rangle}{\|u-x\|} \geq 0\right\}
$$

is the Fréchet subdifferential of $f$ at $x$. Similarly, if $x \in \Omega \subset X$, then

$$
N_{\Omega}^{F}(x):=\left\{x^{*} \in X^{*} \mid \limsup _{u \stackrel{\Omega}{\rightarrow} x} \frac{\left\langle x^{*}, u-x\right\rangle}{\|u-x\|} \leq 0\right\}
$$

is the Fréchet normal cone to $\Omega$ at $x$. The denotation $u \stackrel{\Omega}{\rightarrow} x$ in the last formula means that $u \rightarrow x$ with $u \in \Omega$. In the convex case, sets (1) and (2) reduce to the subdifferential and normal cone in the sense of convex analysis. 


\section{Infinite Collections of Sets}

In this section, we recall the definitions and characterizations of stationarity and regularity properties for a collection of sets $\Omega:=\left\{\Omega_{i}\right\}_{i \in I} \subset X$, where $I$ is an arbitrary index set, possibly infinite, $|I|>1$. The sets are considered near a point $\bar{x} \in \bigcap_{i \in I} \Omega_{i}$.

For full definitions, proofs of the results given in this section and some discussions we address the reader to $[24$, Section 3].

When defining the mentioned properties, we analyze families of subcollections of $\boldsymbol{\Omega}$ corresponding to finite subsets $J \subset I$ and impose restrictions on the cardinality $|J|$ using a given gauge function $\Phi: \mathbb{R}_{+} \rightarrow \mathbb{R}_{+} \cup\{+\infty\}$, which determines the "growth rate" of the cardinality $|J|$ of finite subsets $J \subset I$. Choosing a smaller gauge function leads to a more (less) restrictive stationarity (regularity) property.

To simplify the definitions, we use the following notations:

$$
\begin{gathered}
\mathcal{J}:=\{J \subset I|1<| J \mid<\infty\}, \\
\mathcal{J}_{\alpha}:=\{J \subset I|1<| J \mid<\Phi(\alpha)\} \quad(\alpha>0) .
\end{gathered}
$$

Obviously $\mathcal{J}_{\alpha} \subset \mathcal{J}$ and $\mathcal{J}_{\alpha}=\mathcal{J}$ if $\Phi(\alpha)=\infty$.

Definition 3.1 The collection of sets $\Omega$ is

$\left(\mathrm{AS}_{\Phi}\right)$ approximately $\Phi$-stationary at $\bar{x}$ iff, for any $\varepsilon>0$, there exist $\left.\rho \in\right] 0, \varepsilon[; \alpha \in] 0, \varepsilon\left[; J \in \mathcal{J}_{\alpha} ; \omega_{i} \in \Omega_{i} \cap B_{\varepsilon}(\bar{x})\right.$ and $a_{i} \in X(i \in J)$ such that $\max _{i \in J}\left\|a_{i}\right\|<\alpha \rho$ and

$$
\bigcap_{i \in J}\left(\Omega_{i}-\omega_{i}-a_{i}\right) \bigcap(\rho \mathbb{B})=\emptyset
$$

$\left(\mathrm{UR}_{\Phi}\right)$ uniformly $\Phi$-regular at $\bar{x}$ iff there exists an $\alpha_{0}>0$ and an $\varepsilon>0$ such that

$$
\bigcap_{i \in J}\left(\Omega_{i}-\omega_{i}-a_{i}\right) \bigcap(\rho \mathbb{B}) \neq \emptyset
$$

for any $\alpha \in] 0, \alpha_{0}[; \rho \in] 0, \varepsilon\left[; J \in \mathcal{J}_{\alpha} ; \omega_{i} \in \Omega_{i} \cap B_{\varepsilon}(\bar{x})\right.$, and $a_{i} \in X(i \in J)$ satisfying $\max _{i \in J}\left\|a_{i}\right\| \leq \alpha \rho$;

$\left(\mathrm{FNAS}_{\Phi}\right)$ Fréchet normally approximately $\Phi$-stationary at $\bar{x}$ iff, for any $\varepsilon>0$, there exist $\left.\alpha \in\right] 0, \varepsilon\left[; J \in \mathcal{J}_{\alpha}\right.$; $x_{i} \in \Omega_{i} \cap B_{\varepsilon}(\bar{x})$ and $x_{i}^{*} \in N_{\Omega_{i}}^{F}\left(x_{i}\right)(i \in J)$ such that

$$
\left\|\sum_{i \in J} x_{i}^{*}\right\|<\alpha \sum_{i \in J}\left\|x_{i}^{*}\right\|
$$

$\left(\mathrm{FNUR}_{\Phi}\right)$ Fréchet normally uniformly $\Phi$-regular at $\bar{x}$ iff there exists an $\alpha_{0}>0$ and an $\varepsilon>0$ such that

$$
\left\|\sum_{i \in J} x_{i}^{*}\right\| \geq \alpha \sum_{i \in J}\left\|x_{i}^{*}\right\|
$$

for any $\alpha \in] 0, \alpha_{0}\left[; J \in \mathcal{J}_{\alpha} ; x_{i} \in \Omega_{i} \cap B_{\varepsilon}(\bar{x})\right.$ and $x_{i}^{*} \in N_{\Omega_{i}}^{F}\left(x_{i}\right)(i \in J)$. 
Note that the $\Phi$-regularity properties $\left(\mathrm{UR}_{\Phi}\right)$ and $\left(\mathrm{FNUR}_{\Phi}\right)$ in Definition 3.1 are negations of the corresponding $\Phi$-stationarity properties $\left(\mathrm{AS}_{\Phi}\right)$ and $\left(\mathrm{FNAS}_{\Phi}\right)$, respectively.

The next theorem establishes the relationship between the approximate $\Phi$-stationarity (uniform $\Phi$-regularity) properties. It combines Corollaries 4.5.1 and 4.5.3 in [24].

Theorem 3.1 Let $\bar{x} \in \bigcap_{i \in I} \Omega_{i},|I|>1$. Suppose $X$ is Asplund and the sets $\Omega_{i}(i \in I)$ are locally closed near $\bar{x}$. The collection of sets $\boldsymbol{\Omega}$ is approximately $\Phi$-stationary (uniformly $\Phi$-regular) at $\bar{x}$ if and only if it is Fréchet normally approximately $\Phi$-stationary (Fréchet normally uniformly $\Phi$-regular) at $\bar{x}$.

Moreover, for any $\varepsilon>0$, conditions $\left(A S_{\Phi}\right)$ and $\left(F N A S_{\Phi}\right)\left(\left(U R_{\Phi}\right)\right.$ and $\left.\left(F N U R_{\Phi}\right)\right)$ are satisfied with the same number $\alpha$ and set of indices $J$.

Remark 3.1 The Fréchet normal approximate $\Phi$-stationarity property (FNAS $S_{\Phi}$ ) can be interpreted as a kind of separation property for a collection of sets. Thanks to Theorem 3.1, this separation property is necessary and sufficient for the collection of sets to be approximately $\Phi$-stationary.

Remark 3.2 Defined above $\Phi$-stationarity and $\Phi$-regularity properties depend on the choice of the gauge function $\Phi$. Since Theorem 3.1 establishes the equivalence of the corresponding stationarity or regularity properties with the same gauge function, it guarantees the same "growth rate" of $|J|$ as $\alpha \downarrow 0$ in primal and dual space conditions. When applying Theorem 3.1 for characterizing stationarity (regularity) of a specific collection of sets, it is important to find the smallest (largest) function such that the property in question still holds true. Then the theorem provides the strongest conclusion. Possible choices of $\Phi$ that could be of interest in applications are discussed in [24, Remark 4.6]. In a particular case when $\Phi$ satisfies conditions of the type $\alpha \Phi(\alpha) \rightarrow 0$ as $\alpha \downarrow 0$, property $\left(A S_{\Phi}\right)$ recaptures the concept of "R-perturbed extremal system" while the stationarity part of Theorem 3.1 strengthens the "rated extremal principles" from [19].

Remark 3.3 When dealing with infinite systems, it seems reasonable to consider gauge functions $\Phi$ such that $\Phi(\alpha) \rightarrow \infty$ as $\alpha \downarrow 0$. However, the above definitions of $\Phi$-stationarity and $\Phi$-regularity properties as well as their characterization in the Theorem 3.1 are valid without this requirement.

If $I$ is a finite set, then one can take a constant function $\Phi(\alpha)=|I|+1$ for all $\alpha>0$. The stationarity and regularity properties in the above definitions will coincide with the corresponding conventional properties considered in [29-31].

Remark 3.4 The normal stationarity and regularity properties $\left(F N A S_{\Phi}\right)$ and $\left(F N U R_{\Phi}\right)$ can be extended to general (not necessarily Fréchet) normal cone operators satisfying certain natural properties while preserving their relationship with the properties $\left(A S_{\Phi}\right)$ and $\left(U R_{\Phi}\right)$ stated in Theorem 3.1. We refer the readers to [24] for the exact definitions and characterizations.

In the special case $\Phi \equiv+\infty$, we will omit $\Phi$ in the names and notations of the corresponding properties. It is easy to check that, in this case, one can omit condition $\alpha \in] 0, \varepsilon[$ in the definitions of stationarity properties and 
condition $\alpha \in] 0, \alpha_{0}$ [ in the definitions of regularity properties, replacing $\alpha$ in the rest of these properties by $\varepsilon$ and $\alpha_{0}$, respectively. See [24, Definition 4.1] for the exact formulations.

Such properties can be equivalently defined in terms of certain nonnegative (possibly infinite) constants.

Given a $\rho \in] 0, \infty]$ and a $J \in \mathcal{J}$, we first define the next constant characterizing the mutual arrangement of the finite collection of sets $\left\{\Omega_{i}\right\}_{i \in J}$ in space:

$$
\theta_{\rho}\left[\left\{\Omega_{i}\right\}_{i \in J}\right](\bar{x}):=\sup \left\{r \geq 0 \mid \bigcap_{i \in J}\left(\Omega_{i}-a_{i}\right) \bigcap B_{\rho}(\bar{x}) \neq \emptyset, \forall a_{i} \in r \mathbb{B}\right\}
$$

Then we can define the following constants for the original collection $\boldsymbol{\Omega}$ :

$$
\begin{gathered}
\hat{\theta}[\boldsymbol{\Omega}](\bar{x}):=\sup _{\varepsilon>0} \inf _{\substack{\rho \in] 0, \varepsilon\left[,, J \in \mathcal{J} \\
\omega_{i} \in B_{\varepsilon}(\bar{x}) \cap \Omega_{i}(i \in J)\right.}} \frac{\theta_{\rho}\left[\left\{\Omega_{i}-\omega_{i}\right\}_{i \in J}\right](0)}{\rho}, \\
\hat{\eta}^{F}[\boldsymbol{\Omega}](\bar{x}):=\sup _{\varepsilon>0} \inf _{\substack{J \in \mathcal{J} \\
x_{i} \in \Omega_{i} \cap B_{\varepsilon}(\bar{x}), x_{i}^{*} \in N_{\Omega_{i}}^{F}\left(x_{i}\right)(i \in J) \\
\sum_{i \in J}\left\|x_{i}^{*}\right\|=1}}\left\|\sum_{i \in J} x_{i}^{*}\right\| .
\end{gathered}
$$

If $I$ is a finite set, then constants (3) and (4) reduce to those considered in [29-31].

Proposition 3.1 The collection of sets $\boldsymbol{\Omega}$ is

(AS) approximately stationary at $\bar{x}$ if and only if $\hat{\theta}[\boldsymbol{\Omega}](\bar{x})=0$,

(UR) uniformly regular at $\bar{x}$ if and only if $\hat{\theta}[\boldsymbol{\Omega}](\bar{x})>0$,

(FNAS) Fréchet normally approximately stationary at $\bar{x}$ if and only if $\hat{\eta}^{F}[\boldsymbol{\Omega}](\bar{x})=0$,

(FNUR) Fréchet normally uniformly regular at $\bar{x}$ if and only if $\hat{\eta}^{F}[\boldsymbol{\Omega}](\bar{x})>0$.

When positive, constants (3) and (4) provide quantitative characterizations of the corresponding regularity properties.

The next theorem establishes the relationship between constants (3) and (4), and consequently between the pairs of primal space properties (AS) and (UR), on the one hand, and dual space ones (FNAS) and (FNUR), on the other hand. It is a consequence of Theorem 3.1.

Theorem 3.2 Let $\bar{x} \in \bigcap_{i \in I} \Omega_{i},|I|>1$. Suppose $X$ is Asplund and the sets $\Omega_{i}(i \in I)$ are locally closed near $\bar{x}$. Then $\hat{\theta}[\boldsymbol{\Omega}](\bar{x})=\hat{\eta}^{F}[\boldsymbol{\Omega}](\bar{x})$.

Corollary 3.1 Under the conditions of Theorem 3.2, the collection of sets $\boldsymbol{\Omega}$ is approximately stationary (uniformly regular) at $\bar{x}$ if and only if it is Fréchet normally approximately stationary (Fréchet normally uniformly regular) at $\bar{x}$.

When $|I|<\infty$, the stationarity part of Corollary 3.1 represents the Extended extremal principle [32,33]. (Some earlier formulations of this result can be found in [34-36].) It strengthens the Extremal principle [25, 27] which is generally recognized as one of the corner-stones of the contemporary variational analysis ( [26]). 


\section{Normals to Infinite Intersections}

In this section, we recall a group of results from [24, Section 5] on intersection rules: representing Fréchet normals to intersections of infinite collections of sets via normals to particular sets. Their proofs are based on the application of Theorem 3.1.

In this and subsequent sections, we assume that $X$ is an Asplund space and $I$ is a nonempty set of indices, possibly infinite. From now on, we drop the assumption that $|J|>1$ in the definitions of $\mathcal{J}$ and $\mathcal{J}_{\alpha}$ :

$$
\begin{aligned}
\mathcal{J} & :=\{J \subset I|0<| J \mid<\infty\}, \\
\mathcal{J}_{\alpha} & :=\{J \subset I|0<| J \mid<\Phi(\alpha)\} .
\end{aligned}
$$

Recalling that $\Phi$-stationarity properties, introduced in Definition 3.1, in fact reduce consideration of an infinite collection of sets to that of a family of its finite subcollections, it is clear that techniques based on Theorem 3.1 can be applicable not to arbitrary Fréchet normals to the intersection, but only to those which are "approximately normal" to the intersections of certain finite subsystems.

In the definition below, a gauge function $\Phi: \mathbb{R}_{+} \rightarrow \mathbb{R}_{+} \cup\{+\infty\}$ is used again. Such functions were discussed in the previous section.

Definition 4.1 An element $x^{*} \in X^{*}$ is

- Fréchet $\Phi$-normal to the intersection $\Omega=\bigcap_{i \in I} \Omega_{i}$ at $\bar{x} \in \Omega$ iff, for any $\varepsilon>0$, there exist $\left.\rho>0, \alpha \in\right] 0, \varepsilon[$, and $J \in \mathcal{J}_{\alpha}$ such that

$$
\left\langle x^{*}, x-\bar{x}\right\rangle<\alpha\|x-\bar{x}\| \quad \forall x \in \bigcap_{i \in J} \Omega_{i} \bigcap B_{\rho}(\bar{x}) \backslash\{\bar{x}\}
$$

- Fréchet finitely normal to the intersection $\Omega=\bigcap_{i \in I} \Omega_{i}$ at $\bar{x} \in \Omega$ iff, for any $\varepsilon>0$, there exists a $\rho>0$ and a subset $J \in \mathcal{J}$ such that

$$
\left\langle x^{*}, x-\bar{x}\right\rangle<\varepsilon\|x-\bar{x}\| \quad \forall x \in \bigcap_{i \in J} \Omega_{i} \bigcap B_{\rho}(\bar{x}) \backslash\{\bar{x}\} .
$$

It is immediate from the definition that every Fréchet $\Phi$-normal element to the intersection $\Omega=\bigcap_{i \in I} \Omega_{i}$ is Fréchet finitely normal to this intersection, while every Fréchet finitely normal element is Fréchet normal to $\Omega$ in the sense of definition (2). If the collection is finite and $\Phi(\alpha)>|I|$ for all $\alpha>0$, then every Fréchet normal element to $\Omega$ is automatically Fréchet $\Phi$-normal to the intersection $\Omega=\bigcap_{i \in I} \Omega_{i}$.

The next theorem established in [24] (see [24, Theorem 5.2]) provides a general intersection rule for Fréchet $\Phi$-normal elements to an infinite intersection of sets.

Theorem 4.1 Let $\bar{x} \in \Omega=\bigcap_{i \in I} \Omega_{i}$ and the sets $\Omega_{i}(i \in I)$ are locally closed near $\bar{x}$. If $x^{*} \in X^{*}$ is Fréchet $\Phi$-normal to the intersection $\bigcap_{i \in I} \Omega_{i}$ at $\bar{x}$, then, for any $\varepsilon>0$, there exist $\left.\alpha \in\right] 0, \varepsilon\left[; J \in \mathcal{J}_{\alpha} ; x_{i} \in \Omega_{i} \cap B_{\varepsilon}(\bar{x})\right.$, $x_{i}^{*} \in N_{\Omega_{i}}^{F}\left(x_{i}\right)(i \in J) ;$ and $a \lambda \geq 0$ such that

$$
\sum_{i \in J}\left\|x_{i}^{*}\right\|+\lambda\left\|x^{*}\right\|+2 \lambda=1 \quad \text { and } \quad\left\|\lambda x^{*}-\sum_{i \in J} x_{i}^{*}\right\|<\alpha .
$$


Remark 4.1 Given a neighborhood $U$ of $\bar{x}$, it is sufficient to require in Theorem 4.1 that only sets $\Omega_{i}$ not containing $U$ are closed.

The main feature of the first condition in (5) is that the elements $x_{i}^{*}(i \in J)$ and number $\lambda$ cannot be zero simultaneously. This point is formulated clearer in the conclusion of the next corollary (see [24, Corollary 5.3.1]).

Corollary 4.1 Under the assumptions of Theorem 4.1, for any $\varepsilon>0$, there exist $\alpha \in] 0, \varepsilon\left[; J \in \mathcal{J}_{\alpha} ; x_{i} \in \Omega_{i} \cap\right.$ $B_{\varepsilon}(\bar{x}), x_{i}^{*} \in N_{\Omega_{i}}^{F}\left(x_{i}\right)(i \in J)$; and $a \lambda \geq 0$ such that

$$
\sum_{i \in J}\left\|x_{i}^{*}\right\|+\lambda=1 \quad \text { and }\left\|\lambda x^{*}-\sum_{i \in J} x_{i}^{*}\right\|<c \alpha
$$

where $c:=\left\|x^{*}\right\|+2$.

The number $\alpha$ and set of indices $J$ in conditions in (5) and (6) are related by the growth condition $|J|<\Phi(\alpha)$. If the growth condition is not important, the intersection rule can be formulated in a more conventional way (see [24, Corollary 5.3.2]).

Corollary 4.2 Let $\bar{x} \in \Omega=\bigcap_{i \in I} \Omega_{i}$ and the sets $\Omega_{i}(i \in I)$ are locally closed near $\bar{x}$. If $x^{*} \in X^{*}$ is Fréchet finitely normal to the intersection $\bigcap_{i \in I} \Omega_{i}$ at $\bar{x}$, then, for any $\varepsilon>0$, there exist $J \in \mathcal{J} ; x_{i} \in \Omega_{i} \cap B_{\varepsilon}(\bar{x})$, $x_{i}^{*} \in N_{\Omega_{i}}^{F}\left(x_{i}\right)(i \in J)$; and $a \lambda \geq 0$ such that

$$
\sum_{i \in J}\left\|x_{i}^{*}\right\|+\lambda=1 \quad \text { and }\left\|\lambda x^{*}-\sum_{i \in J} x_{i}^{*}\right\|<\varepsilon
$$

The last corollary generalizes the intersection rules for finite collections of sets (see, e.g., [26, Lemma 3.1], [37, Theorem 2.4]). It also generalizes and strengthens the recent "fuzzy intersection rule for R-normals" in [19, Theorem 5.5].

Note that, strictly speaking, conditions (5), (6), and (7) do not provide representation formulas for $x^{*}$ in terms of $x_{i}^{*}, i \in J$. It is important to have normal versions of these conditions, that is, with $\lambda \neq 0$. To this end, regularity conditions need to be imposed on the collection of sets $\boldsymbol{\Omega}$. The next corollary (see [24, Corollaries 5.3.3 and 5.4.1]) shows that regularity condition (FNUR) acts as a constraint qualification.

Corollary 4.3 Let $\bar{x} \in \Omega=\bigcap_{i \in I} \Omega_{i}$, the sets $\Omega_{i}(i \in I)$ are locally closed near $\bar{x}$ and the collection $\boldsymbol{\Omega}$ is Fréchet normally uniformly regular at $\bar{x}$.

(i) If $x^{*} \in X^{*}$ is Fréchet $\Phi$-normal to the intersection $\bigcap_{i \in I} \Omega_{i}$ at $\bar{x}$, then, for any $\varepsilon>0$ and $\left.\gamma \in\right] 0,1[$, there exist $\alpha \in] 0, \varepsilon\left[; J \in \mathcal{J}_{\alpha} ;\right.$ and $x_{i} \in \Omega_{i} \cap B_{\varepsilon}(\bar{x}), x_{i}^{*} \in N_{\Omega_{i}}^{F}\left(x_{i}\right)(i \in J)$ such that

$$
\left\|x^{*}-\sum_{i \in J} x_{i}^{*}\right\|<c \alpha,
$$

where $c:=\left\|x^{*}\right\|\left(\left(\gamma \hat{\eta}^{F}[\boldsymbol{\Omega}](\bar{x})\right)^{-1}+1\right)+2$. 
(ii) If $x^{*} \in X^{*}$ is Fréchet finitely normal to the intersection $\bigcap_{i \in I} \Omega_{i}$ at $\bar{x}$, then, for any $\varepsilon>0$, there exist $J \in \mathcal{J}$; $x_{i} \in \Omega_{i} \cap B_{\varepsilon}(\bar{x})$ and $x_{i}^{*} \in N_{\Omega_{i}}^{F}\left(x_{i}\right)(i \in J)$ such that

$$
\left\|x^{*}-\sum_{i \in J} x_{i}^{*}\right\|<\varepsilon .
$$

Thanks to Theorem 3.2, the assumption of Fréchet normal uniform regularity of the collection $\boldsymbol{\Omega}$ in Corollary 4.3 can be replaced by the corresponding primal space regularity condition.

\section{Maximum Rule}

An immediate application of the intersection rules formulated above is to developing maximum rules for Fréchet subdifferentials. We are going to consider next the supremum function

$$
f(x):=\sup _{i \in I} f_{i}(x), \quad x \in X
$$

where $I$ is a (possibly infinite) nonempty set of indices and $f_{i}(i \in I)$ are functions from an Asplund space $X$ to the extended real line $\mathbb{R}_{\infty}$.

Recall that the Fréchet subdifferential (1) of $f$ at $x(f(x)<\infty)$ admits an equivalent representation:

$$
\partial^{F} f(x)=\left\{x^{*} \in X^{*} \mid\left(x^{*},-1\right) \in N_{\mathrm{epi} f}^{F}(x, f(x))\right\}
$$

where epi $f=\{(u, \mu) \in X \times \mathbb{R} \mid f(u) \leq \mu\}$ is the epigraph of $f$. It is assumed here that $X \times \mathbb{R}$ is equipped with a norm providing the product topology.

If $\left(x^{*}, \lambda\right) \in N_{\mathrm{epi} f}^{F}(x, \mu)$ for some $\mu \geq f(x)$, then necessarily $\lambda \leq 0$ and $\left(x^{*}, \lambda\right) \in N_{\mathrm{epi} f}^{F}(x, f(x))$. If $\mu>f(x)$, then $\lambda=0$.

The set

$$
\partial^{\infty} f(x):=\left\{x^{*} \in X^{*} \mid\left(x^{*}, 0\right) \in N_{\text {epi } f}^{F}(x, f(x))\right\}
$$

is called the singular Fréchet subdifferential of $f$ at $x$. The two sets (10) and (11) provide complete description of Fréchet normals to epi $f$ at $(x, f(x))$. If $\left(x^{*}, \lambda\right) \in N_{\text {epi } f}^{F}(x, f(x))$, then either $\lambda<0$ and $x^{*} /|\lambda| \in \partial^{F} f(x)$, or $\lambda=0$ and $x^{*} \in \partial^{\infty} f(x)$. If $f(u) \leq f(x)+c\|u-x\|$ for some $c>0$ and all $u \in X$ near $x$ (particularly if $f$ is Lipschitz continuous near $x)$, then $\partial f^{\infty}(x)=\{0\}$.

Fix a point $\bar{x} \in X$ with $f(\bar{x})<\infty$ and an $\varepsilon>0$. Next, we define the set of $\varepsilon$-active indices at $\bar{x}$ :

$$
I_{\varepsilon}(\bar{x}):=\left\{i \in I \mid \sup _{x \in B_{\varepsilon}(\bar{x})} f_{i}(x) \geq f(\bar{x})-\varepsilon\right\} .
$$

The smaller the number $\varepsilon$ is, the smaller the set $I_{\varepsilon}(\bar{x})$ will be.

Let a gauge function $\Phi: \mathbb{R}_{+} \rightarrow \mathbb{R}_{+}$be given. Similarly to the case of the intersection rule, we need to limit ourselves to considering 'finitely generated' Fréchet subgradients of $f$.

Definition 5.1 An element $x^{*} \in X^{*}$ is a 
(i) Fréchet $\Phi$-subgradient of the supremum function (9) at $\bar{x}$ iff, for any $\varepsilon>0$, there exist $\rho>0, \alpha \in] 0, \varepsilon[$ and $J \in \mathcal{J}_{\alpha}$ such that

$$
\sup _{i \in J} f_{i}(x)-f(\bar{x})-\left\langle x^{*}, x-\bar{x}\right\rangle+\alpha\|x-\bar{x}\|>0 \quad \forall x \in B_{\rho}(\bar{x}) \backslash\{\bar{x}\}
$$

(ii) Fréchet finitely generated subgradient of the supremum function (9) at $\bar{x}$ iff, for any $\varepsilon>0$, there exists a $\rho>0$ and a subset $J \in \mathcal{J}$ such that

$$
\sup _{i \in J} f_{i}(x)-f(\bar{x})-\left\langle x^{*}, x-\bar{x}\right\rangle+\varepsilon\|x-\bar{x}\|>0 \quad \forall x \in B_{\rho}(\bar{x}) \backslash\{\bar{x}\}
$$

(iii) singular Fréchet $\Phi$-subgradient of the supremum function $(9)$ at $\bar{x}$ iff $\left(x^{*}, 0\right)$ is Fréchet $\Phi$-normal to the intersection $\bigcap_{i \in I}$ epi $f_{i}$ at $(\bar{x}, f(\bar{x}))$;

(iv) singular Fréchet finitely generated subgradient of the supremum function $(9)$ at $\bar{x}$ iff $\left(x^{*}, 0\right)$ is Fréchet finitely normal to the intersection $\bigcap_{i \in I}$ epi $f_{i}$ at $(\bar{x}, f(\bar{x}))$.

Obviously, every (singular) Fréchet $\Phi$-subgradient of the supremum function (9) is a (singular) Fréchet finitely generated subgradient of this function, while every (singular) Fréchet finitely generated subgradient is a (singular) Fréchet subgradient, that is, it belongs to the (singular) Fréchet subdifferential of $f$. On the other hand, if the index set $I$ is finite and $\Phi(\alpha)>|I|$ for all $\alpha>0$, then every (singular) Fréchet subgradient of $f$ is automatically a (singular) Fréchet $\Phi$-subgradient of the supremum function (9)

The two 'nonsingular' types of subgradients defined in parts (i) and (ii) of Definition 5.1 correspond to 'nonhorizontal' normals to the intersection of epigraphs, namely, (i) (respectively, (ii)) is equivalent to $\left(x^{*},-1\right)$ being Fréchet $\Phi$-normal (respectively, Fréchet finitely normal) to the intersection $\bigcap_{i \in I}$ epi $f_{i}$ at $(\bar{x}, f(\bar{x}))$.

To simplify the statements, we are going to introduce two more notations:

$$
\begin{aligned}
\mathcal{J}_{\varepsilon, \alpha}(\bar{x}) & :=\left\{J \subset I_{\varepsilon}(\bar{x})|0<| J \mid<\Phi(\alpha)\right\}, \\
\mathcal{J}_{\varepsilon}(\bar{x}) & :=\left\{J \subset I_{\varepsilon}(\bar{x})|0<| J \mid<\infty\right\} .
\end{aligned}
$$

It is easy to check that, in the above definition, sets $\mathcal{J}_{\alpha}$ and $\mathcal{J}$ can be replaced by $\mathcal{J}_{\varepsilon, \alpha}(\bar{x})$ and $\mathcal{J}_{\varepsilon}(\bar{x})$, respectively.

In the rest of the section, we assume that $X \times \mathbb{R}$ is equipped with the maximum norm: $\|(x, \mu)\|=\max \{\|x\|,|\mu|\}$. Then the dual norm is of the sum type: $\left\|\left(x^{*}, \lambda\right)\right\|=\left\|x^{*}\right\|+|\lambda|$.

Theorem 5.1 Suppose there exists an $\varepsilon_{0}>0$ such that the functions $f_{i}, i \in I_{\varepsilon_{0}}(\bar{x})$, are lower semicontinuous near $\bar{x}$.

(i) If $x^{*} \in X^{*}$ is a Fréchet $\Phi$-subgradient of the supremum function (9) at $\bar{x}$, then, for any $\varepsilon>0$, there exist $\alpha \in] 0, \varepsilon\left[; \quad J \in \mathcal{J}_{\varepsilon, \alpha}(\bar{x}) ; x_{i} \in B_{\varepsilon}(\bar{x}), x_{i}^{*} \in X^{*}, \lambda_{i} \geq 0(i \in J) ;\right.$ and $a \lambda \geq 0$ such that

$$
f_{i}\left(x_{i}\right) \leq f(\bar{x})+\varepsilon, x_{i}^{*} \in \lambda_{i} \partial^{F} f_{i}\left(x_{i}\right) \text { if } \lambda_{i}>0 \text { and } x_{i}^{*} \in \partial^{\infty} f_{i}\left(x_{i}\right) \text { if } \lambda_{i}=0
$$

and

$$
\sum_{i \in J}\left(\left\|x_{i}^{*}\right\|+\lambda_{i}\right)+\lambda\left\|x^{*}\right\|+3 \lambda=1 \text { and }\left\|\lambda x^{*}-\sum_{i \in J} x_{i}^{*}\right\|+\left|\lambda-\sum_{i \in J} \lambda_{i}\right|<\alpha .
$$


(ii) If $x^{*} \in X^{*}$ is a singular Fréchet $\Phi$-subgradient of the supremum function (9) at $\bar{x}$, then, for any $\varepsilon>0$, there exist $\alpha \in] 0, \varepsilon\left[; \quad J \in \mathcal{J}_{\varepsilon, \alpha}(\bar{x}) ; x_{i} \in B_{\varepsilon}(\bar{x}), x_{i}^{*} \in X^{*}, \lambda_{i} \geq 0\right.$ (i $\left.\in J\right)$ satisfying (13); and a $\lambda \geq 0$ such that

$$
\sum_{i \in J}\left(\left\|x_{i}^{*}\right\|+\lambda_{i}\right)+\lambda\left\|x^{*}\right\|+2 \lambda=1 \quad \text { and }\left\|\lambda x^{*}-\sum_{i \in J} x_{i}^{*}\right\|+\sum_{i \in J} \lambda_{i}<\alpha .
$$

Proof The assertion is a direct consequence of Theorem 4.1. It is sufficient to observe that epi $f=\bigcap_{i \in I}$ epi $f_{i}$. The sets epi $f_{i}, i \in I_{\varepsilon_{0}}(\bar{x})$, are locally closed near $(\bar{x}, f(\bar{x}))$. For $i \notin I_{\varepsilon_{0}}(\bar{x})$, the sets epi $f_{i}$ do not have to be locally closed (Remark 4.1). Such sets can be ignored since the corresponding Fréchet normal elements will be zero.

If $x^{*} \in X^{*}$ is a Fréchet $\Phi$-subgradient of the supremum function $(9)$ at $\bar{x}$, then $\left(x^{*},-1\right)$ is Fréchet $\Phi$-normal to the intersection $\bigcap_{i \in I}$ epi $f_{i}$ at $(\bar{x}, f(\bar{x}))$. Let $\varepsilon>0$ be given. Without any loss of generality, we will assume that $\varepsilon<\varepsilon_{0}$. By Theorem 4.1, there exist $\left.\alpha \in\right] 0, \varepsilon\left[; J \in \mathcal{J}_{\alpha} ;\left(x_{i}, \mu_{i}\right) \in \operatorname{epi} f_{i} \cap B_{\varepsilon}(\bar{x}, f(\bar{x})),\left(x_{i}^{*},-\lambda_{i}\right) \in N_{\text {epi } f_{i}}^{F}\left(x_{i}, \mu_{i}\right)\right.$ $(i \in J) ;$ and a $\lambda \geq 0$ such that

$$
\sum_{i \in J}\left\|\left(x_{i}^{*},-\lambda_{i}\right)\right\|+\lambda\left\|\left(x^{*},-1\right)\right\|+2 \lambda=1 \quad \text { and } \quad\left\|\lambda\left(x^{*},-1\right)-\sum_{i \in J}\left(x_{i}^{*},-\lambda_{i}\right)\right\|<\alpha .
$$

Then $f\left(x_{i}\right) \leq f(\bar{x})+\varepsilon, \lambda_{i} \geq 0$, and $\left(x_{i}^{*},-\lambda_{i}\right) \in N_{\text {epi } f_{i}}^{F}\left(x_{i}, f\left(x_{i}\right)\right)$. If $i \notin I_{\varepsilon_{0}}(\bar{x})$, then $\left(x_{i}, \mu_{i}\right) \in \operatorname{int}$ epi $f_{i}$, and, consequently, $\left\|x_{i}^{*}\right\|=\lambda_{i}=0$. If $\lambda_{i}>0$, then $x_{i}^{*} / \lambda_{i} \in \partial^{F} f_{i}\left(x_{i}\right)$. Otherwise, $x_{i}^{*} \in \partial^{\infty} f_{i}\left(x_{i}\right)$. Conditions (14) follow after observing that $\left\|\left(x^{*},-1\right)\right\|=\left\|x^{*}\right\|+1,\left\|\left(x_{i}^{*},-\lambda_{i}\right)\right\|=\left\|x_{i}^{*}\right\|+\lambda_{i}$, and

$$
\left\|\lambda\left(x^{*},-1\right)-\sum_{i \in J}\left(x_{i}^{*},-\lambda_{i}\right)\right\|=\left\|\left(\lambda x^{*}-\sum_{i \in J} x_{i}^{*},-\lambda+\sum_{i \in J} \lambda_{i}\right)\right\|=\left\|\lambda x^{*}-\sum_{i \in J} x_{i}^{*}\right\|+\left|\lambda-\sum_{i \in J} \lambda_{i}\right| .
$$

Similarly, if $x^{*} \in X^{*}$ is a singular Fréchet $\Phi$-subgradient of the supremum function (9) at $\bar{x}$, then, for any $\varepsilon>0$, there exist $\alpha \in] 0, \varepsilon\left[; J \in \mathcal{J}_{\alpha} ; x_{i} \in B_{\varepsilon}(\bar{x}), x_{i}^{*} \in X^{*}, \lambda_{i} \geq 0(i \in J)\right.$ satisfying (13); and a $\lambda \geq 0$ such that

$$
\sum_{i \in J}\left\|\left(x_{i}^{*},-\lambda_{i}\right)\right\|+\lambda\left\|x^{*}\right\|+2 \lambda=1 \quad \text { and } \quad\left\|\lambda\left(x^{*}, 0\right)-\sum_{i \in J}\left(x_{i}^{*},-\lambda_{i}\right)\right\|<\alpha .
$$

This is equivalent to (15).

The next two corollaries are analogues of Corollaries 4.1 and 4.2 , respectively.

Corollary 5.1 In the conclusions of Theorem 5.1, conditions (14) and (15) can be replaced by

$$
\sum_{i \in J}\left(\left\|x_{i}^{*}\right\|+\lambda_{i}\right)+\lambda=1 \quad \text { and } \quad\left\|\lambda x^{*}-\sum_{i \in J} x_{i}^{*}\right\|+\left|\lambda-\sum_{i \in J} \lambda_{i}\right|<c_{1} \alpha,
$$

and

$$
\sum_{i \in J}\left(\left\|x_{i}^{*}\right\|+\lambda_{i}\right)+\lambda=1 \quad \text { and }\left\|\lambda x^{*}-\sum_{i \in J} x_{i}^{*}\right\|+\sum_{i \in J} \lambda_{i}<c_{2} \alpha,
$$

respectively, where $c_{1}:=\left\|x^{*}\right\|+3$ and $c_{2}:=\left\|x^{*}\right\|+2$.

Corollary 5.2 Suppose there exists an $\varepsilon_{0}>0$ such that the functions $f_{i}, i \in I_{\varepsilon_{0}}(\bar{x})$, are lower semicontinuous near $\bar{x}$. 
(i) If $x^{*} \in X^{*}$ is a Fréchet finitely generated subgradient of the supremum function (9) at $\bar{x}$, then, for any $\varepsilon>0$, there exist $J \in \mathcal{J}_{\varepsilon}(\bar{x}) ; x_{i} \in B_{\varepsilon}(\bar{x}), x_{i}^{*} \in X^{*}, \lambda_{i} \geq 0$ (i $\left.\in J\right)$ satisfying (13); and a $\lambda \geq 0$ such that

$$
\sum_{i \in J}\left(\left\|x_{i}^{*}\right\|+\lambda_{i}\right)+\lambda=1, \quad\left\|\lambda x^{*}-\sum_{i \in J} x_{i}^{*}\right\|<\varepsilon \quad \text { and }\left|\lambda-\sum_{i \in J} \lambda_{i}\right|<\varepsilon .
$$

(ii) If $x^{*} \in X^{*}$ is a singular Fréchet finitely generated subgradient of the supremum function (9) at $\bar{x}$, then, for any $\varepsilon>0$, there exist $J \in \mathcal{J}_{\varepsilon}(\bar{x}) ; x_{i} \in B_{\varepsilon}(\bar{x}), x_{i}^{*} \in X^{*}, \lambda_{i} \geq 0$ (i $\left.\in J\right)$ satisfying (13); and a $\lambda \geq 0$ such that

$$
\sum_{i \in J}\left(\left\|x_{i}^{*}\right\|+\lambda_{i}\right)+\lambda=1, \quad\left\|\lambda x^{*}-\sum_{i \in J} x_{i}^{*}\right\|<\varepsilon \quad \text { and } \sum_{i \in J} \lambda_{i}<\varepsilon .
$$

Normal versions (that is, with $\lambda \neq 0$ ) of conditions (14)-(19) can be formulated under certain regularity requirements imposed on the collection of functions $\mathcal{F}:=\left\{f_{i}\right\}_{i \in I}$. All stationarity and regularity properties of collections of sets in Definition 3.1 produce corresponding properties of collections of functions when applied to associated collections of their epigraphs. Here, we need a single regularity property related to the following constant which represents an analogue of constant (4):

$$
\hat{\eta}^{F}[\mathcal{F}](\bar{x}):=\sup _{\varepsilon>0} \inf _{\substack{J \in \mathcal{J}_{\varepsilon}(\bar{x}) \\\left(x_{i}, \mu_{i}\right) \in \operatorname{epi} f_{i} \cap B_{\varepsilon}(\bar{x}, f(\bar{x})),\left(x_{i}^{*},-\lambda_{i}\right) \in N_{\text {epi } f_{i}}^{F}\left(x_{i}, \mu_{i}\right)(i \in J) \\ \sum_{i \in J}\left\|x_{i}^{*}\right\|=1}}\left(\left\|\sum_{i \in J} x_{i}^{*}\right\|+\sum_{i \in J} \lambda_{i}\right) .
$$

Definition 5.2 The collection of functions $\mathcal{F}$ is Fréchet normally uniformly regular at $\bar{x}$ iff $\hat{\eta}[\mathcal{F}](\bar{x})>0$, that is, there exists an $\alpha>0$ and an $\varepsilon>0$ such that

$$
\left\|\sum_{i \in J} x_{i}^{*}\right\|+\sum_{i \in J} \lambda_{i} \geq \alpha \sum_{i \in J}\left\|x_{i}^{*}\right\|
$$

for any $J \in \mathcal{J}_{\varepsilon}(\bar{x}) ;\left(x_{i}, \mu_{i}\right) \in \operatorname{epi} f_{i} \cap B_{\varepsilon}(\bar{x}, f(\bar{x}))$ and $\left(x_{i}^{*},-\lambda_{i}\right) \in N_{\text {epi } f_{i}}^{F}\left(x_{i}, \mu_{i}\right)(i \in J)$.

This condition is automatically satisfied if the functions $f_{i}, i \in I_{\varepsilon}(\bar{x})$, are uniformly Lipschitz near $\bar{x}$. Indeed, if, for each $i \in I_{\varepsilon}(\bar{x}), f_{i}$ is Lipschitz continuous in $B_{\varepsilon}(\bar{x})$ with modulus $l$, then $N_{\text {epi } f_{i}}^{F}\left(x_{i}, \mu_{i}\right)=\{0,0\}$ if $\mu_{i}>f_{i}\left(x_{i}\right)$ and $\left\|x_{i}^{*}\right\| \leq l \lambda_{i}$ if $\left(x_{i}^{*},-\lambda_{i}\right) \in N_{\text {epi } f_{i}}^{F}\left(x_{i}, f_{i}\left(x_{i}\right)\right)$. Thus, for any $J \subset I_{\varepsilon}(\bar{x}) ;\left(x_{i}, \mu_{i}\right) \in$ epi $f_{i} \cap B_{\varepsilon}(\bar{x}, f(\bar{x}))$ and $\left(x_{i}^{*},-\lambda_{i}\right) \in N_{\text {epi } f_{i}}^{F}\left(x_{i}, \mu_{i}\right)(i \in J)$, it holds

$$
\sum_{i \in J}\left\|x_{i}^{*}\right\| \leq l \sum_{i \in J} \lambda_{i} \leq l\left(\left\|\sum_{i \in J} x_{i}^{*}\right\|+\sum_{i \in J} \lambda_{i}\right) .
$$

Hence, (21) holds true with $\alpha:=l^{-1}$.

Condition (21) is also satisfied if a stronger inequality holds true with some $\alpha \in] 0,1]$ :

$$
\left\|\sum_{i \in J} x_{i}^{*}\right\| \geq \alpha \sum_{i \in J}\left\|x_{i}^{*}\right\| .
$$

The next corollary is an analogues of Corollary 4.3. Its conclusions do not contain $\lambda$ and provide characterizations of $x^{*}$ in terms of Fréchet normals to the epigraphs of the functions $f_{i}$.

Corollary 5.3 Suppose there exists an $\varepsilon_{0}>0$ such that the functions $f_{i}, i \in I_{\varepsilon_{0}}(\bar{x})$, are lower semicontinuous near $\bar{x}$, and the collection $\mathcal{F}$ is Fréchet normally uniformly regular at $\bar{x}$. 
(i) If $x^{*} \in X^{*}$ is a Fréchet $\Phi$-subgradient of the supremum function (9) at $\bar{x}$, then, for any $\varepsilon>0$ and $\left.\gamma \in\right] 0,1[$, there exist $\alpha \in] 0, \varepsilon\left[; J \in \mathcal{J}_{\varepsilon, \alpha}(\bar{x}) ; x_{i} \in B_{\varepsilon}(\bar{x}), x_{i}^{*} \in X^{*}\right.$, and $\lambda_{i} \geq 0$ (i $\left.\in J\right)$ satisfying (13), such that

$$
\left\|x^{*}-\sum_{i \in J} x_{i}^{*}\right\|+\left|1-\sum_{i \in J} \lambda_{i}\right|<c \alpha,
$$

where $c:=\left(\left\|x^{*}\right\|+1\right)\left(\gamma \hat{\eta}^{F}[\mathcal{F}](\bar{x})\right)^{-1}+\left\|x^{*}\right\|+3$.

(ii) If $x^{*} \in X^{*}$ is a singular Fréchet $\Phi$-subgradient of the supremum function (9) at $\bar{x}$, then, for any $\varepsilon>0$ and $\gamma \in] 0,1[$, there exist $\alpha \in] 0, \varepsilon\left[; J \in \mathcal{J}_{\varepsilon, \alpha}(\bar{x}) ; x_{i} \in B_{\varepsilon}(\bar{x}), x_{i}^{*} \in X^{*}\right.$, and $\lambda_{i} \geq 0$ (i $\left.\in J\right)$ satisfying (13), such that

$$
\left\|x^{*}-\sum_{i \in J} x_{i}^{*}\right\|+\sum_{i \in J} \lambda_{i}<c \alpha,
$$

where $c:=\left\|x^{*}\right\|\left(\gamma \hat{\eta}^{F}[\mathcal{F}](\bar{x})\right)^{-1}+\left\|x^{*}\right\|+2$.

(iii) If $x^{*} \in X^{*}$ is a Fréchet finitely generated subgradient of the supremum function (9) at $\bar{x}$, then, for any $\varepsilon>0$, there exist $J \in \mathcal{J}_{\varepsilon}(\bar{x}) ; x_{i} \in B_{\varepsilon}(\bar{x}), x_{i}^{*} \in X^{*}$, and $\lambda_{i} \geq 0$ (i $\left.\in J\right)$ satisfying (13), such that

$$
\left\|x^{*}-\sum_{i \in J} x_{i}^{*}\right\|<\varepsilon \quad \text { and } \sum_{i \in J} \lambda_{i}=1 .
$$

(iv) If $x^{*} \in X^{*}$ is a singular Fréchet finitely generated subgradient of the supremum function (9) at $\bar{x}$, then, for any $\varepsilon>0$, there exist $J \in \mathcal{J}_{\varepsilon}(\bar{x}) ; x_{i} \in B_{\varepsilon}(\bar{x}), x_{i}^{*} \in X^{*}$, and $\lambda_{i} \geq 0$ (i $\left.\in J\right)$ satisfying (13), such that

$$
\left\|x^{*}-\sum_{i \in J} x_{i}^{*}\right\|<\varepsilon \quad \text { and } \sum_{i \in J} \lambda_{i}<\varepsilon .
$$

Proof The first two assertions are pretty straightforward. Assertion (iv) follows directly from (ii). Next we are going to prove (iii). If $x^{*} \in X^{*}$ is a Fréchet finitely generated subgradient of the supremum function (9) at $\bar{x}$, then direct application of (i) produces the following assertion: for any $\xi>0$, there exist $J \in \mathcal{J}_{\xi}(\bar{x}) ; x_{i} \in B_{\xi}(\bar{x})$, $x_{i}^{*} \in X^{*}$, and $\lambda_{i} \geq 0(i \in J)$ satisfying (13), such that

$$
\left\|x^{*}-\sum_{i \in J} x_{i}^{*}\right\|+\left|1-\sum_{i \in J} \lambda_{i}\right|<\xi .
$$

Given an $\varepsilon>0$, take a sufficiently small $\xi \in] 0,1\left[\right.$ such that $\xi\left(\left\|x^{*}\right\|+1\right) /(1-\xi)<\varepsilon$. It follows from $(23)$ that

$$
\left\|x^{*}-\sum_{i \in J} x_{i}^{*}\right\|<\xi, \quad\left\|\sum_{i \in J} x_{i}^{*}\right\|<\left\|x^{*}\right\|+\xi, \quad\left|1-\sum_{i \in J} \lambda_{i}\right|<\xi, \quad \sum_{i \in J} \lambda_{i}>1-\xi .
$$

Denote $\gamma:=\sum_{i \in J} \lambda_{i} ; \tilde{\lambda}_{i}:=\lambda_{i} / \gamma, \tilde{x}_{i}^{*}:=x_{i}^{*} / \gamma(i \in J)$. Then $\sum_{i \in J} \tilde{\lambda}_{i}=1$ and

$$
\left\|x^{*}-\sum_{i \in J} \tilde{x}_{i}^{*}\right\|<\left|\gamma^{-1}-1\right|\left\|\sum_{i \in J} x_{i}^{*}\right\|+\xi<\frac{\xi\left(\left\|x^{*}\right\|+\xi\right)}{\gamma}+\xi<\frac{\xi\left(\left\|x^{*}\right\|+1\right)}{1-\xi}<\varepsilon .
$$

Hence, replacing $\tilde{\lambda}_{i}^{*}$ and $\tilde{x}_{i}^{*}$ with $\lambda_{i}^{*}$ and $x_{i}^{*}(i \in J)$, we conclude that conditions (13) and (22) are satisfied.

In the Lipschitz case, Corollary 5.3 (iii) takes a simpler form. 
Corollary 5.4 Suppose there exists an $\varepsilon_{0}>0$ such that the functions $f_{i}, i \in I_{\varepsilon_{0}}(\bar{x})$, are uniformly Lipschitz near $\bar{x}$. If $x^{*} \in X^{*}$ is a Fréchet finitely generated subgradient of the supremum function (9) at $\bar{x}$, then, for any $\varepsilon>0$, there exist $J \in \mathcal{J}_{\varepsilon}(\bar{x}) ; x_{i} \in B_{\varepsilon}(\bar{x}), x_{i}^{*} \in \partial^{F} f_{i}\left(x_{i}\right)$, and $\lambda_{i} \geq 0$ (i $\left.\in J\right)$ such that

$$
\left\|x^{*}-\sum_{i \in J} \lambda_{i} x_{i}^{*}\right\|<\varepsilon \quad \text { and } \sum_{i \in J} \lambda_{i}=1 .
$$

Remark 5.1 When the space is Banach and the functions are convex, a similar result in terms of Fenchel subdifferentials can be established using [38, Theorem 1] and the Brøndsted-Rockafellar theorem.

\section{Optimality Conditions}

Another possible application of the theory developed in the preceding sections is to deducing necessary optimality/stationarity conditions. In this section $X$ is assumed Asplund.

\subsection{Minimax problems}

First, we consider a minimax problem: minimizing the supremum function (9).

$$
\text { Minimize } f(x):=\sup _{i \in I} f_{i}(x) .
$$

Recall that the supremum in (24) is over a possibly infinite nonempty index set $I$.

Let a point $\bar{x} \in X$ with $f(\bar{x})<\infty$ and a gauge function $\Phi: \mathbb{R}_{+} \rightarrow \mathbb{R}_{+}$be given.

Definition 6.1 A point $\bar{x} \in X$ is

(i) Fréchet $\Phi$-stationary for problem (24) iff, for any $\varepsilon>0$, there exist $\rho>0 ; \alpha \in] 0, \varepsilon\left[; J \in \mathcal{J}_{\alpha}\right.$ such that

$$
\sup _{i \in J} f_{i}(x)-f(\bar{x})+\alpha\|x-\bar{x}\|>0 \quad \forall x \in B_{\rho}(\bar{x}) \backslash\{\bar{x}\}
$$

(ii) Fréchet finitely stationary for problem (24) iff, for any $\varepsilon>0$, there exists a $\rho>0$ and a subset $J \in \mathcal{J}$ such that

$$
\sup _{i \in J} f_{i}(x)-f(\bar{x})+\varepsilon\|x-\bar{x}\|>0 \quad \forall x \in B_{\rho}(\bar{x}) \backslash\{\bar{x}\} .
$$

Obviously, every Fréchet $\Phi$-stationary point is Fréchet finitely stationary, while every Fréchet finitely stationary point $\bar{x}$ is stationary for $f$ in the sense that

$$
\liminf _{x \rightarrow \bar{x}} \frac{f(x)-f(\bar{x})}{\|x-\bar{x}\|} \geq 0
$$

(or, equivalently, that $0 \in \partial^{F} f(\bar{x})$.) If the index set $I$ is finite and $\Phi(\alpha)>|I|$ for all $\alpha>0$, then every stationary point of $f$, in particular, every locally minimal point, is Fréchet $\Phi$-stationary. If $|I|=\infty$, then a minimal point does not have to be finitely stationary, unless some additional assumptions are imposed. 
Example 6.1 Let $f_{i}(x)=-\sqrt{|x|} / i$ where $x \in \mathbb{R}$ and $i \in I=\{1,2, \ldots\}$. Then $f(x)=\sup _{i \in I} f_{i}(x)=0$ for any $x \in \mathbb{R}$. Hence, $\bar{x}=0$ is a point of minimum of $f$. Take any nonempty subset $J \in \mathcal{J}$ and denote by $i_{0}$ the maximal number in $J$. Then, for any $\rho>0$, taking an $x \in] 0, \min \left\{i_{0}^{-2}, \rho\right\}[$, we have

$$
\sup _{i \in J} f_{i}(x)+|x|=-\sqrt{x}\left(i_{0}^{-1}-\sqrt{x}\right)<0
$$

which is a violation of (25) with $\varepsilon=1$. Hence, $\bar{x}=0$ is not finitely stationary.

The two stationarity concepts for problem (24) are defined above as purely primal space concepts. At the same time, comparing Definitions 6.1 and 5.1, one can easily see that they admit simple dual space characterizations.

Proposition 6.1 A point $\bar{x} \in X$ is Fréchet $\Phi$-stationary (finitely stationary) for problem (24) if and only if $0_{X^{*}}$ is a Fréchet $\Phi$-subgradient (finitely generated subgradient) of the supremum function (9) at $\bar{x}$.

In the next theorem, which is a direct consequence of Theorem 5.1 (i), Corollary 5.2 (i), and Proposition 6.1, $I_{\varepsilon}(\bar{x})$ denotes the set $(12)$ of $\varepsilon$-active indices at $\bar{x}$.

Theorem 6.1 Suppose there exists an $\varepsilon_{0}>0$ such that the functions $f_{i}, i \in I_{\varepsilon_{0}}(\bar{x})$, are lower semicontinuous near $\bar{x}$.

(i) If $\bar{x}$ is a Fréchet $\Phi$-stationary point for problem (24), then, for any $\varepsilon>0$, there exist $\alpha \in] 0, \varepsilon\left[; J \in \mathcal{J}_{\varepsilon, \alpha}(\bar{x})\right.$; $x_{i} \in B_{\varepsilon}(\bar{x}), x_{i}^{*} \in X^{*}, \lambda_{i} \geq 0(i \in J)$ satisfying (13); and $a \lambda \geq 0$ such that

$$
\sum_{i \in J}\left(\left\|x_{i}^{*}\right\|+\lambda_{i}\right)+3 \lambda=1 \quad \text { and } \quad\left\|\sum_{i \in J} x_{i}^{*}\right\|+\left|\lambda-\sum_{i \in J} \lambda_{i}\right|<\alpha .
$$

(ii) If $\bar{x}$ is a Fréchet finitely stationary point for problem (24), then, for any $\varepsilon>0$, there exist $J \in \mathcal{J}_{\varepsilon}(\bar{x})$; $x_{i} \in B_{\varepsilon}(\bar{x}), x_{i}^{*} \in X^{*}, \lambda_{i} \geq 0(i \in J)$ satisfying (13); and $a \lambda \geq 0$ such that

$$
\sum_{i \in J}\left(\left\|x_{i}^{*}\right\|+\lambda_{i}\right)+\lambda=1, \quad\left\|\sum_{i \in J} x_{i}^{*}\right\|<\varepsilon \quad \text { and }\left|\lambda-\sum_{i \in J} \lambda_{i}\right|<\varepsilon .
$$

The next statement provides necessary stationarity conditions in the normal form. It is a consequence of Corollaries 5.3 (i),(iii) and 5.4.

Corollary 6.1 Suppose there exists an $\varepsilon_{0}>0$ such that the functions $f_{i}, i \in I_{\varepsilon_{0}}(\bar{x})$, are lower semicontinuous near $\bar{x}$, and the collection $\mathcal{F}$ is Fréchet normally uniformly regular at $\bar{x}$.

(i) If $\bar{x}$ is a Fréchet $\Phi$-stationary point for problem (24), then, for any $\varepsilon>0$ and $\gamma \in] 0,1[$, there exist $\alpha \in] 0, \varepsilon[$; $J \in \mathcal{J}_{\varepsilon, \alpha}(\bar{x}) ; x_{i} \in B_{\varepsilon}(\bar{x}), x_{i}^{*} \in X^{*}$, and $\lambda_{i} \geq 0$ (i $\left.i \in J\right)$ satisfying (13), such that

$$
\left\|\sum_{i \in J} x_{i}^{*}\right\|+\left|1-\sum_{i \in J} \lambda_{i}\right|<c \alpha
$$

where $c:=\left(\gamma \hat{\eta}^{F}[\mathcal{F}](\bar{x})\right)^{-1}+3$. 
(ii) If $\bar{x}$ is a Fréchet finitely stationary point for problem (24), then, for any $\varepsilon>0$, there exist $J \in \mathcal{J}_{\varepsilon}(\bar{x})$; $x_{i} \in B_{\varepsilon}(\bar{x}), x_{i}^{*} \in X^{*}$, and $\lambda_{i} \geq 0(i \in J)$ satisfying (13), such that

$$
\left\|\sum_{i \in J} x_{i}^{*}\right\|<\varepsilon \quad \text { and } \quad \sum_{i \in J} \lambda_{i}=1 .
$$

Moreover, if the functions $f_{i}, i \in I_{\varepsilon_{0}}(\bar{x})$, are uniformly Lipschitz near $\bar{x}$ then, for any $\varepsilon>0$, there exist $J \in \mathcal{J}_{\varepsilon}(\bar{x}) ; x_{i} \in B_{\varepsilon}(\bar{x}), x_{i}^{*} \in \partial^{F} f_{i}\left(x_{i}\right)$, and $\lambda_{i} \geq 0(i \in J)$ such that

$$
\left\|\sum_{i \in J} \lambda_{i} x_{i}^{*}\right\|<\varepsilon \quad \text { and } \sum_{i \in J} \lambda_{i}=1 .
$$

\subsection{Constrained optimization: set constraints}

Next, we consider a constrained optimization problem with constraints given by arbitrary sets:

$$
\text { Minimize } f(x) \text { subject to } x \in \bigcap_{i \in I} \Omega_{i}
$$

where $f: X \rightarrow \mathbb{R}_{\infty}, f(\bar{x})<\infty, \bar{x} \in \Omega_{i} \subset X(i \in I)$, and the index set $I$ can be infinite.

This problem can be treated as a particular case of the minimax problem (24). Indeed, assuming without any loss of generality that $0 \notin I$, set $\tilde{f}_{0}=f-f(\bar{x}), \tilde{f}_{i}=\delta_{\Omega_{i}}$ - the indicator function of $\Omega_{i}$, and $\tilde{I}=I \cup\{0\}$. Then $\bar{x}$ is a local solution to (26) if and only if it is a local minimum point of the function

$$
\tilde{f}(x):=\sup _{i \in \tilde{I}} \tilde{f}_{i}(x), \quad x \in X
$$

The next definition introduces two stationarity concepts for problem (26), which can be considered as direct analogues of the corresponding stationarity concepts for problem (24) from Definition 6.1.

Definition 6.2 A point $\bar{x} \in X$ is

(i) Fréchet $\Phi$-stationary for problem (26) iff, for any $\varepsilon>0$, there exist $\rho>0, \alpha \in] 0, \varepsilon\left[\right.$, and $J \in \mathcal{J}_{\alpha}$ such that

$$
f(x)-f(\bar{x})+\alpha\|x-\bar{x}\|>0 \quad \forall x \in \bigcap_{i \in J} \Omega_{i} \bigcap B_{\rho}(\bar{x}) \backslash\{\bar{x}\}
$$

(ii) Fréchet finitely stationary for problem (26) iff, for any $\varepsilon>0$, there exists a $\rho>0$ and a subset $J \in \mathcal{J}$ such that

$$
f(x)-f(\bar{x})+\varepsilon\|x-\bar{x}\|>0 \quad \forall x \in \bigcap_{i \in J} \Omega_{i} \bigcap B_{\rho}(\bar{x}) \backslash\{\bar{x}\} .
$$

Application of Theorem 6.1 to the problem of minimizing (27) leads to the following statement.

Theorem 6.2 Suppose $f$ is lower semicontinuous and the sets $\Omega_{i}(i \in I)$ are locally closed near $\bar{x}$. 
(i) If $\bar{x}$ is a Fréchet $\Phi$-stationary point for problem (26), then, for any $\varepsilon>0$, there exist $\alpha \in] 0, \varepsilon\left[; J \in \mathcal{J}_{\alpha}\right.$; $x_{i} \in B_{\varepsilon}(\bar{x}), x_{i}^{*} \in X^{*}(i \in J \cup\{0\}) ;$ and $a \lambda_{0} \geq 0$ such that

$$
\begin{gathered}
f\left(x_{0}\right) \leq f(\bar{x})+\varepsilon, x_{0}^{*} \in \lambda_{0} \partial^{F} f\left(x_{0}\right) \text { if } \lambda_{0}>0 \text { and } x_{0}^{*} \in \partial^{\infty} f\left(x_{0}\right) \text { if } \lambda_{0}=0, \\
x_{i} \in \Omega_{i}, \quad x_{i}^{*} \in N_{\Omega_{i}}^{F}\left(x_{i}\right), \quad(i \in J) \\
\sum_{i \in J \cup\{0\}}\left\|x_{i}^{*}\right\|+\lambda_{0} \leq 1 \text { and }\left\|\sum_{i \in J \cup\{0\}} x_{i}^{*}\right\|+\frac{1}{3}\left(1-\sum_{i \in J \cup\{0\}}\left\|x_{i}^{*}\right\|-4 \lambda_{0}\right)<\alpha .
\end{gathered}
$$

(ii) If $\bar{x}$ is a Fréchet finitely stationary point for problem (26), then, for any $\varepsilon>0$, there exist $J \in \mathcal{J} ; x_{i} \in B_{\varepsilon}(\bar{x})$, $x_{i}^{*} \in X^{*}(i \in J \cup\{0\})$ and $a \lambda_{0} \geq 0$, satisfying (28) and (29), and such that

$$
\sum_{i \in J \cup\{0\}}\left\|x_{i}^{*}\right\|+\lambda_{0} \leq 1,\left\|\sum_{i \in J \cup\{0\}} x_{i}^{*}\right\|<\varepsilon \text { and }\left(1-\sum_{i \in J \cup\{0\}}\left\|x_{i}^{*}\right\|-2 \lambda_{0}\right)_{+}<\varepsilon .
$$

Proof Let $\bar{x}$ be a Fréchet $\Phi$-stationary point for problem (26) and an $\varepsilon>0$ be given. Then $\bar{x}$ is a Fréchet $\Phi$-stationary point for the problem of minimizing $(27)$ and, by Theorem 6.1 , there exist $\alpha \in] 0, \varepsilon[; \tilde{J} \subset \tilde{I},|\tilde{J}|<\Phi(\alpha)$; $x_{i} \in B_{\varepsilon}(\bar{x}), x_{i}^{*} \in X^{*}, \lambda_{i} \geq 0(i \in \tilde{J}) ;$ and a $\lambda \geq 0$ such that

$$
\begin{aligned}
\tilde{f}_{i}\left(x_{i}\right) \leq & \tilde{f}(\bar{x})+\varepsilon, x_{i}^{*} \in \lambda_{i} \partial^{F} \tilde{f}_{i}\left(x_{i}\right) \text { if } \lambda_{i}>0 \text { and } x_{i}^{*} \in \partial^{\infty} \tilde{f}_{i}\left(x_{i}\right) \text { if } \lambda_{i}=0(i \in \tilde{J}), \\
& \sum_{i \in \tilde{J}}\left(\left\|x_{i}^{*}\right\|+\lambda_{i}\right)+3 \lambda=1 \text { and }\left\|\sum_{i \in \tilde{J}} x_{i}^{*}\right\|+\left|\lambda-\sum_{i \in \tilde{J}} \lambda_{i}\right|<\alpha .
\end{aligned}
$$

Put $J=\tilde{J} \backslash\{0\}$. Obviously, $|J|<\Phi(\alpha)$. Without any loss of generality, we can assume that $0 \in \tilde{J}$ : otherwise, we can always add $x_{0}=\bar{x}, x_{0}^{*}=0$, and $\lambda_{0}=0$. If $i \in J$, then necessarily $x_{i} \in \Omega_{i}$ and $\partial^{F} \tilde{f}_{i}\left(x_{i}\right)=\partial^{\infty} \tilde{f}_{i}\left(x_{i}\right)=N_{\Omega_{i}}^{F}\left(x_{i}\right)$. Hence, $\tilde{J}=J \cup\{0\}$ and conditions (31) take the form of (28) and (29). Conditions (32) do not impose many restrictions on numbers $\lambda$ and $\lambda_{i}, i \in J$. The sum $\sum_{i \in J} \lambda_{i}$, which is present in both conditions in (32), can be replaced by a single nonnegative variable $\tilde{\lambda}$ :

$$
\sum_{i \in \tilde{J}}\left\|x_{i}^{*}\right\|+\lambda_{0}+\tilde{\lambda}+3 \lambda=1 \text { and }\left\|\sum_{i \in \tilde{J}} x_{i}^{*}\right\|+\left|\lambda-\left(\lambda_{0}+\tilde{\lambda}\right)\right|<\alpha .
$$

If conditions (33) are also satisfied with some nonnegative $\lambda$ and $\tilde{\lambda}$, then they are satisfied with particular numbers minimizing the left-hand side of the inequality in (33) under the given equality constraint (and with fixed $x_{i}^{*}$, $i \in \tilde{J}$, and $\left.\lambda_{0}\right)$ :

$$
\lambda=\min \left(\frac{1-\sum_{i \in \tilde{J}}\left\|x_{i}^{*}\right\|-\lambda_{0}}{3}, \frac{1-\sum_{i \in \tilde{J}}\left\|x_{i}^{*}\right\|}{4}\right) \text { and } \tilde{\lambda}=\left(\frac{1-\sum_{i \in \tilde{J}}\left\|x_{i}^{*}\right\|}{4}-\lambda_{0}\right)_{+} .
$$

With these numbers we have

$$
\left|\lambda-\left(\lambda_{0}+\tilde{\lambda}\right)\right|=\frac{1}{3}\left(1-\sum_{i \in \tilde{J}}\left\|x_{i}^{*}\right\|-4 \lambda_{0}\right)_{+} .
$$

Hence, conditions (33) imply (30).

The proof of the second statement goes along the same lines, with conditions (32) replaced by the following ones:

$$
\sum_{i \in \tilde{J}}\left(\left\|x_{i}^{*}\right\|+\lambda_{i}\right)+\lambda=1, \quad\left\|\sum_{i \in \tilde{J}} x_{i}^{*}\right\|<\varepsilon \quad \text { and } \quad\left|\lambda-\sum_{i \in \tilde{J}} \lambda_{i}\right|<\varepsilon .
$$


To formulate the normal version of the stationarity conditions, that is, with $\lambda_{0} \neq 0$, certain combined regularity conditions must be imposed on $f$ and the collection of sets $\boldsymbol{\Omega}:=\left\{\Omega_{i}\right\}_{i \in I}$. Such conditions can be formulated using the following analogue of constant (20):

$$
\hat{\eta}^{F}[f, \boldsymbol{\Omega}](\bar{x}):=\sup _{\varepsilon>0} \inf _{\substack{J \in \mathcal{J} \\\left(x_{0}, \mu_{0}\right) \in \operatorname{epi~} f \cap B_{\varepsilon}(\bar{x}, f(\bar{x})) \\\left(x_{0}^{*},-\lambda_{0}\right) \in N_{\text {ep }}^{F} f\left(x_{0}, \mu_{0}\right) \\ x_{i} \in \Omega_{i} \cap B_{\varepsilon}(\bar{x}), x_{i}^{*} \in N_{\Omega_{i}}^{F}\left(x_{i}\right)(i \in J) \\ \sum_{i \in J \cup\{0\}}\left\|x_{i}^{*}\right\|=1}}\left(\left\|\sum_{i \in J \cup\{0\}}^{*}\right\|+\lambda_{0}\right) .
$$

Definition 6.3 The couple $\{f, \boldsymbol{\Omega}\}$ is Fréchet normally uniformly regular at $\bar{x}$ iff $\hat{\eta}[f, \boldsymbol{\Omega}](\bar{x})>0$, that is, there exists an $\alpha>0$ and an $\varepsilon>0$ such that

$$
\left\|\sum_{i \in J \cup\{0\}} x_{i}^{*}\right\|+\lambda_{0} \geq \alpha \sum_{i \in J \cup\{0\}}\left\|x_{i}^{*}\right\|
$$

for any $J \in \mathcal{J} ;\left(x_{0}, \mu_{0}\right) \in$ epi $f \cap B_{\varepsilon}(\bar{x}, f(\bar{x})),\left(x_{0}^{*},-\lambda_{0}\right) \in N_{\text {epi } f}^{F}\left(x_{0}, \mu_{0}\right) ; x_{i} \in \Omega_{i} \cap B_{\varepsilon}(\bar{x})$ and $x_{i}^{*} \in N_{\Omega_{i}}^{F}\left(x_{i}\right)$ $(i \in J)$.

Corollary 6.2 Suppose $f$ is lower semicontinuous, the sets $\Omega_{i}(i \in I)$ are locally closed near $\bar{x}$, and the couple $\{f, \boldsymbol{\Omega}\}$ is Fréchet normally uniformly regular at $\bar{x}$.

(i) If $\bar{x}$ is a Fréchet $\Phi$-stationary point for problem (26), then, for any $\varepsilon>0$ and $\gamma \in] 0,1[$, there exist $\alpha \in] 0, \varepsilon[$; $J \in \mathcal{J}_{\alpha} ; x_{i} \in B_{\varepsilon}(\bar{x})$ and $x_{i}^{*} \in X^{*}(i \in J \cup\{0\})$ such that

$$
f\left(x_{0}\right) \leq f(\bar{x})+\varepsilon, \quad x_{0}^{*} \in \partial^{F} f\left(x_{0}\right) ; \quad x_{i} \in \Omega_{i}, \quad x_{i}^{*} \in N_{\Omega_{i}}^{F}\left(x_{i}\right) \quad(i \in J)
$$

and

$$
\left\|\sum_{i \in J \cup\{0\}} x_{i}^{*}\right\|<c \alpha,
$$

where $c:=\left(2+\hat{\eta}^{F}[f, \boldsymbol{\Omega}](\bar{x})\right) /\left[\gamma \min \left\{\hat{\eta}^{F}[f, \boldsymbol{\Omega}](\bar{x}), 1 / 3\right\}\right]$.

(ii) If $\bar{x}$ is a Fréchet finitely stationary point for problem (26), then, for any $\varepsilon>0$, there exist $J \in \mathcal{J} ; x_{i} \in B_{\varepsilon}(\bar{x})$ and $x_{i}^{*} \in X^{*}(i \in J \cup\{0\})$ satisfying (34), such that

$$
\left\|\sum_{i \in J \cup\{0\}} x_{i}^{*}\right\|<\varepsilon .
$$

Proof Let $\bar{x}$ be a Fréchet $\Phi$-stationary point for problem (26) and let $\varepsilon>0$ and $\left.\gamma^{\prime} \in\right] \gamma, 1[$ be given. Since the couple $\{f, \boldsymbol{\Omega}\}$ is Fréchet normally uniformly regular at $\bar{x}$, by Definition 6.3 , setting $\alpha_{1}:=\gamma^{\prime} \min \left\{\hat{\eta}^{F}[f, \boldsymbol{\Omega}](\bar{x}), 1 / 3\right\}$, there exists a $\delta>0$ such that

$$
\left\|\sum_{i \in J \cup\{0\}} x_{i}^{*}\right\|+\lambda_{0} \geq \alpha_{1} \sum_{i \in J \cup\{0\}}\left\|x_{i}^{*}\right\|
$$

for any $J \in \mathcal{J} ;\left(x_{0}, \mu_{0}\right) \in$ epi $f \cap B_{\delta}(\bar{x}, f(\bar{x})),\left(x_{0}^{*},-\lambda_{0}\right) \in N_{\text {epi } f}^{F}\left(x_{0}, \mu_{0}\right) ; x_{i} \in \Omega_{i} \cap B_{\delta}(\bar{x})$ and $x_{i}^{*} \in N_{\Omega_{i}}^{F}\left(x_{i}\right)$ $(i \in J)$. 
By Theorem 6.2, there exist $\left.\alpha \in] 0, \min \left\{\varepsilon,\left(1-\gamma / \gamma^{\prime}\right) \alpha_{1}\right\}\right) ; J \in \mathcal{J}_{\alpha} ; x_{i} \in B_{\min \{\varepsilon, \delta\}}(\bar{x}), x_{i}^{*} \in X^{*}(i \in J \cup\{0\})$ and a $\lambda_{0} \geq 0$ satisfying (28) and (29) such that (30) holds true. (30) and (35) imply the following estimates:

$$
\begin{aligned}
\left(2+\alpha_{1}\right) \lambda_{0}>\frac{1}{3}-\left(\frac{1}{3}-\alpha_{1}\right)\left(\sum_{i \in J \cup\{0\}}\left\|x_{i}^{*}\right\|\right. & \left.+\lambda_{0}\right)-\alpha \geq \alpha_{1}-\alpha>\left(\gamma / \gamma^{\prime}\right) \alpha_{1} \\
& =\gamma \min \left\{\hat{\eta}^{F}[f, \boldsymbol{\Omega}](\bar{x}), 1 / 3\right\}=\left(2+\hat{\eta}^{F}[f, \boldsymbol{\Omega}](\bar{x})\right) / c \geq\left(2+\alpha_{1}\right) / c
\end{aligned}
$$

The conclusion of the first assertion follows after dividing the second inequality in (30) by $\lambda_{0}$ and replacing $\lambda_{0}^{-1} x_{i}^{*}$ with $x_{i}^{*}$.

The second assertion is a consequence of the first one.

The conclusions of Corollary 6.2 can be rewritten in a more conventional way.

Corollary 6.3 Suppose $f$ is lower semicontinuous, the sets $\Omega_{i}(i \in I)$ are locally closed near $\bar{x}$, and the couple $\{f, \boldsymbol{\Omega}\}$ is Fréchet normally uniformly regular at $\bar{x}$.

(i) If $\bar{x}$ is a Fréchet $\Phi$-stationary point for problem (26), then, for any $\gamma \in] 0,1[$,

$$
\begin{aligned}
0 \in \bigcap_{\varepsilon>0} \bigcup\left\{\partial^{F} f\left(x_{0}\right)+\sum_{i \in J} N_{\Omega_{i}}^{F}\left(x_{i}\right)+c \alpha \mathbb{B}^{*} \mid x_{0} \in B_{\varepsilon}(\bar{x}),\right. & f\left(x_{0}\right) \leq f(\bar{x})+\varepsilon ; \\
& \alpha \in] 0, \varepsilon\left[; \quad J \in \mathcal{J}_{\alpha} ; x_{i} \in \Omega_{i} \cap B_{\varepsilon}(\bar{x})(i \in J)\right\}
\end{aligned}
$$

where $c:=\left(2+\hat{\eta}^{F}[f, \boldsymbol{\Omega}](\bar{x})\right) /\left[\gamma \min \left\{\hat{\eta}^{F}[f, \boldsymbol{\Omega}](\bar{x}), 1 / 3\right\}\right]$.

(ii) If $\bar{x}$ is a Fréchet finitely stationary point for problem (26), then

$$
\begin{aligned}
0 \in \bigcap_{\varepsilon>0} \bigcup\left\{\partial^{F} f\left(x_{0}\right)+\sum_{i \in J} N_{\Omega_{i}}^{F}\left(x_{i}\right)+\varepsilon \mathbb{B}^{*} \mid x_{0} \in B_{\varepsilon}(\bar{x}), f\left(x_{0}\right) \leq f(\bar{x})+\varepsilon\right. & \\
& \left.J \in \mathcal{J} ; x_{i} \in \Omega_{i} \cap B_{\varepsilon}(\bar{x})(i \in J)\right\} .
\end{aligned}
$$

Remark 6.1 Theorem 6.2 and its corollaries give necessary conditions of Fréchet $\Phi$-stationarity or finite stationarity. They are not automatically applicable to arbitrary stationary or even minimal points unless $|I|<\infty$. This fact reflects the intrinsic complexity of infinite programming problems which require a variety of meaningful concepts of stationarity. To formulate necessary optimality conditions on the base of Theorem 6.2, one must ensure that the solution of the optimization problem under consideration satisfies one of the stationarity properties in Definition 6.2. For instance, if $\bar{x}$ is a local solution to problem (26), one can require that the following two conditions are satisfied:

1) the approximate Fréchet zero sum rule holds true at $\bar{x}$ for the sum of two functions: $f$ and $\delta_{\Omega}$, where $\Omega:=\bigcap_{i \in I} \Omega_{i}$

2) every Fréchet normal element to $\Omega$ at $\bar{x}$ is Fréchet $\Phi$-normal to the intersection $\bigcap_{i \in I} \Omega_{i}$ at $\bar{x}$.

Conditions of this type were used in [19, Theorem 5.15]. The second part of this theorem is a direct consequence of Corollary 6.3 (ii) above. When $|I|<\infty$, Theorem 6.2 and its corollaries provide standard approximate (fuzzy) optimality conditions - see the limiting form of these conditions in [39, Corollary 5.6]. 


\subsection{Constrained optimization: inequality constraints}

In this subsection, we consider a constrained optimization problem with constrains given by inequalities:

$$
\text { Minimize } f_{0}(x) \text { subject to } f_{i}(x) \leq 0, i \in I \text {, }
$$

where $f_{i}: X \rightarrow \mathbb{R}_{\infty}(i \in I)$ and the index set $I$ can be infinite. To avoid confusion, we are assuming that $0 \notin I$.

Obviously, (36) is a particular case of problem (26) with $\Omega_{i}=\left\{x \in X \mid f_{i}(x) \leq 0\right\}$. However, it seems more convenient to interpret solutions to (36) as those of a minimax problem of the type (24).

Let $\bar{x}$ be a local solution to $(36)$ with $f_{0}(\bar{x})<\infty$. Without any loss of generality, we will assume that $f_{0}(\bar{x})=0$. Then $\bar{x}$ is a local minimal point of the function

$$
f(x):=\sup _{i \in I \cup\{0\}} f_{i}(x), \quad x \in X .
$$

Let a gauge function $\Phi: \mathbb{R}_{+} \rightarrow \mathbb{R}_{+}$be given.

Definition 6.4 A point $\bar{x} \in X$ is

(i) Fréchet $\Phi$-stationary for problem (36) iff, for any $\varepsilon>0$, there exist $\rho>0, \alpha \in] 0, \varepsilon\left[\right.$, and $J \in \mathcal{J}_{\alpha}$ such that

$$
\sup _{i \in J \cup\{0\}} f_{i}(x)+\alpha\|x-\bar{x}\|>0 \quad \forall x \in B_{\rho}(\bar{x}) \backslash\{\bar{x}\}
$$

(ii) Fréchet finitely stationary for problem (36) iff, for any $\varepsilon>0$, there exists a $\rho>0$ and a subset $J \in \mathcal{J}$ such that

$$
\sup _{i \in J \cup\{0\}} f_{i}(x)+\varepsilon\|x-\bar{x}\|>0 \quad \forall x \in B_{\rho}(\bar{x}) \backslash\{\bar{x}\} .
$$

Obviously, every Fréchet $\Phi$-stationary point is Fréchet finitely stationary, while every Fréchet finitely stationary point $\bar{x}$ is stationary for the function $f$ given by (37).

The next two statements are realizations of Theorem 6.1 and Corollary 6.1 , respectively. $I_{\varepsilon}(\bar{x})$ denotes the set (12) of $\varepsilon$-active indices at $\bar{x}$.

Theorem 6.3 Suppose there exists an $\varepsilon_{0}>0$ such that the functions $f_{i}, i \in I_{\varepsilon_{0}}(\bar{x}) \cup\{0\}$, are lower semicontinuous near $\bar{x}$.

(i) If $\bar{x}$ is a Fréchet $\Phi$-stationary point for problem (36), then, for any $\varepsilon>0$, there exist $\alpha \in] 0, \varepsilon\left[; J \in \mathcal{J}_{\varepsilon, \alpha}(\bar{x})\right.$; $x_{i} \in B_{\varepsilon}(\bar{x}), x_{i}^{*} \in X^{*}, \lambda_{i} \geq 0(i \in J \cup\{0\})$ satisfying (13); and a $\lambda \geq 0$ such that

$$
\sum_{i \in J \cup\{0\}}\left(\left\|x_{i}^{*}\right\|+\lambda_{i}\right)+3 \lambda=1 \quad \text { and }\left\|\sum_{i \in J \cup\{0\}} x_{i}^{*}\right\|+\left|\lambda-\sum_{i \in J \cup\{0\}} \lambda_{i}\right|<\alpha .
$$

(ii) If $\bar{x}$ is a Fréchet finitely stationary point for problem (36), then, for any $\varepsilon>0$, there exist $J \in \mathcal{J}_{\varepsilon}(\bar{x})$; $x_{i} \in B_{\varepsilon}(\bar{x}), x_{i}^{*} \in X^{*}, \lambda_{i} \geq 0(i \in J \cup\{0\})$ satisfying (13); and $a \lambda \geq 0$ such that

$$
\sum_{i \in J \cup\{0\}}\left(\left\|x_{i}^{*}\right\|+\lambda_{i}\right)+\lambda=1, \quad\left\|\sum_{i \in J \cup\{0\}} x_{i}^{*}\right\|<\varepsilon \quad \text { and }\left|\lambda-\sum_{i \in J \cup\{0\}} \lambda_{i}\right|<\varepsilon .
$$


Corollary 6.4 Suppose there exists an $\varepsilon_{0}>0$ such that the functions $f_{i}, i \in I_{\varepsilon_{0}}(\bar{x}) \cup\{0\}$, are lower semicontinuous near $\bar{x}$, and the collection $\left\{f_{i}\right\}_{i \in I \cup\{0\}}$ is Fréchet normally uniformly regular at $\bar{x}$.

(i) If $\bar{x}$ is a Fréchet $\Phi$-stationary point for problem (36), then, for any $\varepsilon>0$ and $\gamma \in] 0,1[$, there exist $\alpha \in] 0, \varepsilon[$; $J \in \mathcal{J}_{\varepsilon, \alpha}(\bar{x}) ; x_{i} \in B_{\varepsilon}(\bar{x}), x_{i}^{*} \in X^{*}$, and $\lambda_{i} \geq 0$ (i $\left.\in J \cup\{0\}\right)$ satisfying (13), such that

$$
\left\|\sum_{i \in J \cup\{0\}} x_{i}^{*}\right\|+\left|1-\sum_{i \in J \cup\{0\}} \lambda_{i}\right|<c \alpha,
$$

where $c:=\left(\gamma \hat{\eta}^{F}\left[\left\{f_{i}\right\}_{i \in I \cup\{0\}}\right](\bar{x})\right)^{-1}+3$.

(ii) If $\bar{x}$ is a Fréchet finitely stationary point for problem (36), then, for any $\varepsilon>0$, there exist $J \in \mathcal{J}_{\varepsilon}(\bar{x})$; $x_{i} \in B_{\varepsilon}(\bar{x}), x_{i}^{*} \in X^{*}$, and $\lambda_{i} \geq 0(i \in J \cup\{0\})$ satisfying (13), such that

$$
\left\|\sum_{i \in J \cup\{0\}} x_{i}^{*}\right\|<\varepsilon \quad \text { and } \sum_{i \in J \cup\{0\}} \lambda_{i}=1 .
$$

Moreover, if the functions $f_{i}, i \in I_{\varepsilon_{0}}(\bar{x}) \cup\{0\}$, are uniformly Lipschitz near $\bar{x}$, then, for any $\varepsilon>0$, there exist $J \in \mathcal{J}_{\varepsilon}(\bar{x}) ; x_{i} \in B_{\varepsilon}(\bar{x}), x_{i}^{*} \in \partial^{F} f_{i}\left(x_{i}\right)$, and $\lambda_{i} \geq 0(i \in J \cup\{0\})$ such that

$$
\left\|\sum_{i \in J \cup\{0\}} \lambda_{i} x_{i}^{*}\right\|<\varepsilon \quad \text { and } \sum_{i \in J \cup\{0\}} \lambda_{i}=1 .
$$

When $|I|<\infty$, the conclusions of Corollary 6.4 (ii) are pretty standard, cf. [39, Subsection 5.1.3]. As an illustration of the above results, the next corollary presents the conclusions of Corollary 6.4 (ii) in a more conventional way.

Corollary 6.5 Suppose there exists an $\varepsilon_{0}>0$ such that the functions $f_{i}, i \in I_{\varepsilon_{0}}(\bar{x}) \cup\{0\}$, are lower semicontinuous near $\bar{x}$, and the collection $\left\{f_{i}\right\}_{i \in I \cup\{0\}}$ is Fréchet normally uniformly regular at $\bar{x}$. If $\bar{x}$ is a Fréchet finitely stationary point for problem (36), then

$$
\begin{aligned}
0 \in \bigcap_{\varepsilon>0} \bigcup\left\{\sum_{i \in J \cup\{0\}} \lambda_{i} \partial^{F} f_{i}\left(x_{i}\right)+\varepsilon \mathbb{B}^{*} \mid\right. & J \in \mathcal{J}_{\varepsilon} ; \\
& \left.x_{i} \in B_{\varepsilon}(\bar{x}), f_{i}\left(x_{i}\right) \leq f(\bar{x})+\varepsilon, \lambda_{i} \geq 0(i \in J \cup\{0\}) ; \sum_{i \in J \cup\{0\}} \lambda_{i}=1\right\}
\end{aligned}
$$

with the convention that $0 \cdot \partial^{F} f_{i}\left(x_{i}\right)=\partial^{\infty} f_{i}\left(x_{i}\right)$.

To ensure the normal form of the above stationarity conditions, certain constraint qualifications are required. They can be defined using the next regularity constant (Recall that the set $\mathcal{F}:=\left\{f_{i}\right\}_{i \in I}$ involves only the functions defining the constraints of the problem):

$$
\begin{aligned}
& \hat{\zeta}[\mathcal{F}](\bar{x}):=\sup _{\varepsilon>0} \inf _{\substack{J \in \mathcal{J}_{\varepsilon}(\bar{x}) \\
\left(x_{i}, \mu_{i}\right) \in \operatorname{epi} f_{i} \cap B_{\varepsilon}(\bar{x}, 0),\left(x_{i}^{*},-\lambda_{i}\right) \in N_{\mathrm{epi} f_{i}}^{F}\left(x_{i}, \mu_{i}\right)(i \in J)}}\left\|\sum_{i \in J} x_{i}^{*}\right\| . \\
& \sum_{i \in J} \lambda_{i}=1
\end{aligned}
$$

Definition 6.5 The Fréchet normal constraint qualification is satisfied for problem $(36)$ at $\bar{x}$ iff $\hat{\zeta}[\mathcal{F}](\bar{x})>0$, that is, there exists an $\alpha>0$ and an $\varepsilon>0$ such that

$$
\left\|\sum_{i \in J} x_{i}^{*}\right\| \geq \alpha \sum_{i \in J} \lambda_{i}
$$

for any $J \in \mathcal{J}_{\varepsilon}(\bar{x}) ;\left(x_{i}, \mu_{i}\right) \in$ epi $f_{i} \cap B_{\varepsilon}(\bar{x}, 0)$ and $\left(x_{i}^{*},-\lambda_{i}\right) \in N_{\text {epi } f_{i}}^{F}\left(x_{i}, \mu_{i}\right)(i \in J)$. 
Corollary 6.6 Suppose there exists an $\varepsilon_{0}>0$ such that the functions $f_{i}, i \in I_{\varepsilon_{0}}(\bar{x}) \cup\{0\}$, are lower semicontinuous near $\bar{x}$, the collection $\left\{f_{i}\right\}_{i \in I \cup\{0\}}$ is Fréchet normally uniformly regular at $\bar{x}$, and the Fréchet normal constraint qualification is satisfied for problem (36) at $\bar{x}$.

(i) If $\bar{x}$ is a Fréchet $\Phi$-stationary point for problem (36), then, for any $\varepsilon>0$ and $\gamma \in] 0,1[$, there exist $\alpha \in] 0, \varepsilon[$; $J \in \mathcal{J}_{\varepsilon, \alpha}(\bar{x}) ; x_{i} \in B_{\varepsilon}(\bar{x}), x_{i}^{*} \in X^{*}$, and $\lambda_{i} \geq 0$ (i $\left.\in J \cup\{0\}\right)$ satisfying (13), such that (38) holds true with $c:=\left(\gamma \hat{\eta}^{F}[\mathcal{F}](\bar{x})\right)^{-1}+3$ and

$$
\left\|x_{0}^{*}\right\|+\lambda_{0}>\gamma \min \left\{\hat{\zeta}^{F}[\mathcal{F}](\bar{x}), 1\right\}
$$

(ii) If $\bar{x}$ is a Fréchet finitely stationary point for problem (36), then, for any $\varepsilon>0$ and $\gamma \in] 0,1[$, there exist $J \in \mathcal{J}_{\varepsilon}(\bar{x}) ; x_{i} \in B_{\varepsilon}(\bar{x}), x_{i}^{*} \in X^{*}$, and $\lambda_{i} \geq 0$ (i $\left.\in J \cup\{0\}\right)$ satisfying (13), such that (39) and (40) hold true. Moreover, if the functions $f_{i}, i \in I_{\varepsilon_{0}}(\bar{x}) \cup\{0\}$, are uniformly Lipschitz near $\bar{x}$, then, for any $\varepsilon>0$, there exist $J \in \mathcal{J}_{\varepsilon}(\bar{x}) ; x_{i} \in B_{\varepsilon}(\bar{x}), x_{i}^{*} \in \partial^{F} f_{i}\left(x_{i}\right)(i \in J \cup\{0\}) ;$ and $\lambda_{i} \geq 0$ (i $\left.i \in J\right)$ such that

$$
\left\|x_{0}^{*}+\sum_{i \in J} \lambda_{i} x_{i}^{*}\right\|<\varepsilon .
$$

Proof Let $\bar{x}$ be a Fréchet $\Phi$-stationary point for problem (36) and let $\varepsilon>0$ and $\gamma \in] 0,1[$ be given. Chose a $\left.\gamma^{\prime} \in\right] \gamma, 1[$. Since the Fréchet normal constraint qualification is satisfied for problem (36) at $\bar{x}$, by Definition 6.5, setting $\alpha_{1}:=\gamma^{\prime} \min \left\{\hat{\zeta}^{F}[\mathcal{F}](\bar{x}), 1\right\}$, there exists a $\delta>0$ such that

$$
\left\|\sum_{i \in J} x_{i}^{*}\right\| \geq \alpha_{1} \sum_{i \in J} \lambda_{i}
$$

for any $J \in \mathcal{J}_{\varepsilon}(\bar{x}) ;\left(x_{i}, \mu_{i}\right) \in$ epi $f_{i} \cap B_{\delta}(\bar{x}, 0)$ and $\left(x_{i}^{*},-\lambda_{i}\right) \in N_{\text {epi } f_{i}}^{F}\left(x_{i}, \mu_{i}\right)(i \in J)$.

By Corollary 6.4, setting $c:=\left(\gamma \hat{\eta}^{F}\left[\left\{f_{i}\right\}_{i \in I \cup\{0\}}\right](\bar{x})\right)^{-1}+3$, there exist $\left.\alpha \in\right] 0, \varepsilon\left[\right.$ satisfying $c \alpha<\left(\gamma^{\prime}-\right.$ $\gamma) \min \left\{\hat{\zeta}^{F}[\mathcal{F}](\bar{x}), 1\right\} ; J \in \mathcal{J}_{\varepsilon, \alpha}(\bar{x}) ; x_{i} \in B_{\min \{\varepsilon, \delta\}}(\bar{x}), x_{i}^{*} \in X^{*}$, and $\lambda_{i} \geq 0(i \in J \cup\{0\})$ satisfying (13), such that (38) holds true. (38) and (41) imply the following inequality:

$$
\alpha_{1} \sum_{i \in J} \lambda_{i}+\left|1-\sum_{i \in J} \lambda_{i}\right|<\left\|x_{0}^{*}\right\|+\lambda_{0}+c \alpha,
$$

and, consequently,

$$
1-\left(1-\alpha_{1}\right) \sum_{i \in J} \lambda_{i}<\left\|x_{0}^{*}\right\|+\lambda_{0}+c \alpha \quad \text { and } \quad \alpha_{1} \sum_{i \in J} \lambda_{i}<\left\|x_{0}^{*}\right\|+\lambda_{0}+c \alpha .
$$

Eliminating $\sum_{i \in J} \lambda_{i}$ from the above system of inequalities, we obtain the next estimate:

$$
\left\|x_{0}^{*}\right\|+\lambda_{0}>\alpha_{1}-c \alpha>\gamma \min \left\{\hat{\zeta}^{F}[\mathcal{F}](\bar{x}), 1\right\}
$$

Estimate (40) remains valid in the case of a Fréchet finitely stationary point.

Let the functions $f_{i}, i \in I_{\varepsilon_{0}}(\bar{x}) \cup\{0\}$, be uniformly Lipschitz near $\bar{x}$ with modulus $l$ and an $\varepsilon>0$ be given. By Corollary 6.4, there exist $J \in \mathcal{J}_{\varepsilon}(\bar{x}) ; x_{i} \in B_{\varepsilon}(\bar{x}), x_{i}^{*} \in \partial^{F} f_{i}\left(x_{i}\right)$, and $\lambda_{i} \geq 0(i \in J \cup\{0\})$ such that

$$
\left\|\sum_{i \in J \cup\{0\}} \lambda_{i} x_{i}^{*}\right\|<c \varepsilon,
$$


where $c:=(1 / 2) \min \left\{\hat{\zeta}^{F}[\mathcal{F}](\bar{x}), 1\right\} /(l+1)$. Besides, $\tilde{x}_{0}^{*}:=\lambda_{0} x_{0}^{*} \in \lambda_{0} \partial^{F} f_{i}\left(x_{i}\right)$ and $\left\|x_{0}^{*}\right\| \leq l$. Without any loss of generality, condition (40) holds true with $\tilde{x}_{0}^{*}$ and $\gamma=1 / 2$. Then $\lambda_{0}>c$. The conclusion follows after dividing (42) by $\lambda_{0}$ and replacing $\lambda_{i} / \lambda_{0}$ by $\lambda_{i}, i \in J \cup\{0\}$.

\section{Concluding Remarks}

In this article, we demonstrate how the stationarity and regularity criteria of infinite collections of sets developed in [24] can be successfully applied to problems of infinite and semi-infinite programming. We consider several settings of optimization problems which involve (explicitly or implicitly) infinite collections of sets. New definitions of stationarity are introduced. Necessary conditions characterizing stationarity in terms of dual space elements normals and/or subdifferentials - are established. This is achieved by applying the intersection rules from Section 4 to developing maximum rules for Fréchet subdifferentials.

\section{References}

1. Auslender, A., Goberna, M.A., López, M.A.: Penalty and smoothing methods for convex semi-infinite programming. Math. Oper. Res. 34(2), 303-319 (2009).

2. Cánovas, M.J., Dontchev, A.L., López, M.A., Parra, J.: Metric regularity of semi-infinite constraint systems. Math. Program., Ser. B 104(2-3), 329-346 (2005).

3. Cánovas, M.J., Hantoute, A., López, M.A., Parra, J.: Stability of indices in the KKT conditions and metric regularity in convex semi-infinite optimization. J. Optim. Theory Appl. 139(3), 485-500 (2008).

4. Cánovas, M.J., Hantoute, A., López, M.A., Parra, J.: Lipschitz modulus in convex semi-infinite optimization via dc functions. ESAIM Control Optim. Calc. Var. 15(4), 763-781 (2009).

5. Cánovas, M.J., Klatte, D., López, M.A., Parra, J.: Metric regularity in convex semi-infinite optimization under canonical perturbations. SIAM J. Optim. 18(3), 717-732 (2007).

6. Cánovas, M.J., López, M.A., Mordukhovich, B.S., Parra, J.: Variational analysis in semi-infinite and infinite programming, I: Stability of linear inequality systems of feasible solutions. SIAM J. Optim. 20, 1504-1526 (2009).

7. Cánovas, M.J., López, M.A., Mordukhovich, B.S., Parra, J.: Variational analysis in semi-infinite and infinite programming, II: Necessary optimality conditions. SIAM J. Optim. 20(6), 2788-2806 (2010).

8. Chuong, T.D., Huy, N.Q., Yao, J.C.: Subdifferentials of marginal functions in semi-infinite programming. SIAM J. Optim. 20(3), 1462-1477 (2009).

9. Dinh, N., Goberna, M.A., López, M.A., Son, T.Q.: New Farkas-type constraint qualifications in convex infinite programming. ESAIM Control Optim. Calc. Var. 13(3), 580-597 (2007).

10. Goberna, M.A., López, M.A.: Linear Semi-Infinite Optimization, Wiley Series in Mathematical Methods in Practice, vol. 2. John Wiley \& Sons Ltd., Chichester (1998).

11. Goberna, M.A., Terlaky, T., Todorov, M.I.: Sensitivity analysis in linear semi-infinite programming via partitions. Math. Oper. Res. 35(1), 14-26 (2010).

12. Guerra-Vázquez, F., Jongen, H.T., Shikhman, V.: General semi-infinite programming: symmetric MangasarianFromovitz constraint qualification and the closure of the feasible set. SIAM J. Optim. 20(5), 2487-2503 (2010).

13. Hantoute, A., López, M.A.: A complete characterization of the subdifferential set of the supremum of an arbitrary family of convex functions. J. Convex Anal. 15(4), 831-858 (2008). 
14. Hantoute, A., López, M.A., Zălinescu, C.: Subdifferential calculus rules in convex analysis: a unifying approach via pointwise supremum functions. SIAM J. Optim. 19(2), 863-882 (2008).

15. Jongen, H.T., Shikhman, V.: Generalized semi-infinite programming: the nonsmooth symmetric reduction ansatz. SIAM J. Optim. 21(1), 193-211 (2011).

16. Kanzi, N.: Necessary optimality conditions for nonsmooth semi-infinite programming problems. J. Global Optim. 49(4), $713-725(2011)$.

17. Kanzi, N., Nobakhtian, S.: Optimality conditions for non-smooth semi-infinite programming. Optimization 59(5-6), $717-727$ (2010).

18. Kostyukova, O.I., Tchemisova, T.V., Yermalinskaya, S.A.: Convex semi-infinite programming: implicit optimality criterion based on the concept of immobile indices. J. Optim. Theory Appl. 145(2), 325-342 (2010).

19. Mordukhovich, B.S., Phan, H.M.: Rated extremal principles for finite and infinite systems. Optimization 60(7), 893-923 (2011).

20. Mordukhovich, B.S., Phan, H.M.: Tangential extremal principles for finite and infinite systems of sets, II: Applications to semi-infinite and multiobjective optimization. Math. Program. (2012). To be published

21. Rückmann, J.J., Shapiro, A.: Augmented Lagrangians in semi-infinite programming. Math. Program. 116(1-2, Ser. B), 499-512 (2009).

22. Shapiro, A.: Semi-infinite programming, duality, discretization and optimality conditions. Optimization 58(2), 133-161 (2009).

23. Zheng, X.Y., Yang, X.: Lagrange multipliers in nonsmooth semi-infinite optimization problems. Math. Oper. Res. 32(1), 168-181 (2007).

24. Kruger, A.Y., López, M.A.: Stationarity and regularity of infinite collections of sets. J. Optim. Theory Appl. 154(2) (2012) DOI 10.1007/s10957-012-0043-4.

25. Kruger, A.Y., Mordukhovich, B.S.: Extremal points and the Euler equation in nonsmooth optimization problems. Dokl. Akad. Nauk BSSR 24(8), 684-687, 763 (1980). In Russian

26. Mordukhovich, B.S.: Variational Analysis and Generalized Differentiation. I: Basic Theory, Grundlehren der Mathematischen Wissenschaften [Fundamental Principles of Mathematical Sciences], vol. 330. Springer-Verlag, Berlin (2006).

27. Mordukhovich, B.S., Shao, Y.: Differential characterizations of covering, metric regularity, and Lipschitzian properties of multifunctions between Banach spaces. Nonlinear Anal. 25(12), 1401-1424 (1995).

28. Rockafellar, R.T., Wets, R.J.B.: Variational Analysis, Grundlehren der Mathematischen Wissenschaften [Fundamental Principles of Mathematical Sciences], vol. 317. Springer-Verlag, Berlin (1998).

29. Kruger, A.Y.: Stationarity and regularity of set systems. Pac. J. Optim. 1(1), 101-126 (2005).

30. Kruger, A.Y.: About regularity of collections of sets. Set-Valued Anal. 14(2), 187-206 (2006).

31. Kruger, A.Y.: About stationarity and regularity in variational analysis. Taiwanese J. Math. 13(6A), 1737-1785 (2009).

32. Kruger, A.Y.: On Fréchet subdifferentials. J. Math. Sci. 116(3), 3325-3358 (2003).

33. Kruger, A.Y.: Weak stationarity: eliminating the gap between necessary and sufficient conditions. Optimization 53(2), $147-164$ (2004).

34. Kruger, A.Y.: On the extremality of set systems. Dokl. Nats. Akad. Nauk Belarusi 42(1), 24-28, 123 (1998). In Russian

35. Kruger, A.Y.: Strict $(\varepsilon, \delta)$-semidifferentials and the extremality of sets and functions. Dokl. Nats. Akad. Nauk Belarusi 44(2), 19-22, 122 (2000). In Russian

36. Kruger, A.Y.: Strict $(\varepsilon, \delta)$-subdifferentials and extremality conditions. Optimization 51(3), 539-554 (2002).

37. Wang, B., Wang, D.: On the fuzzy intersection rule. Nonlinear Anal. 75(3), 1623-1634 (2012).

38. López, M.A., Volle, M.: On the subdifferential of the supremum of an arbitrary family of extended real-valued functions. Rev. R. Acad. Cienc. Exactas Fís. Nat. Ser. A Math. RASCAM 105(1), 3-21 (2011).

39. Mordukhovich, B.S.: Variational Analysis and Generalized Differentiation. II: Applications, Grundlehren der Mathematischen Wissenschaften [Fundamental Principles of Mathematical Sciences], vol. 331. Springer-Verlag, Berlin (2006). 